\title{
Disegno sempre anche quando penso. I luoghi e l'architettura attraverso visioni inedite di Cascarano
}

\author{
Antonio Conte \\ Ivana Passamani
}

Abstract

"Disegno sempre anche quando penso". II contributo tenta un difficile compito: presentare in uno spazio ridotto l'esperienza, l'attività creativa, poetica e tecnica di un architetto, per andare oltre la semplice catalogazione delle opere. Michele Cascarano vive disegnando e disegna vivendo luoghi, città e architetture, testimoniando un pensiero teorico. Indagare su questa figura silenziosa consente di aprire uno spaccato su alcuni aspetti della storia della professione di architetto nel secondo Novecento. II contributo vuole evidenziare i caratteri e le questioni metodologiche dell'intreccio fittissimo tra fondamenti teorici e ricerca in architettura, attraverso la sua rappresentazione: disegni, acquerelli che fissano sulla carta città visitate e immaginate, tra memoria e oblio di opere senza tempo. II lavoro di Cascarano mostra l'esistenza di una ricerca individuale intesa come opera collettiva dell'essere architetto, che ripone nell'arte del disegno un'originale esperienza della realtà e del mondo. Da questo fondamento egli trae l'energia e la forza rappresentativa per disegnare architetture tendenziose, collocate tra rilievo, memoria e progetto. Così l'atto del fabbricare è l'opera d'arte della creazione architettonica, e configura un mondo abitabile non soggetto alle rovine del tempo e all'incuria degli uomini. L'osservatore viene coinvolto nell'awentura vertiginosa di un pensiero: il disegno di mosaici di città è il nesso, il senso di esistenza. Pensare disegnando, per comprendere alcune realtà del mondo e renderle ideali [I].

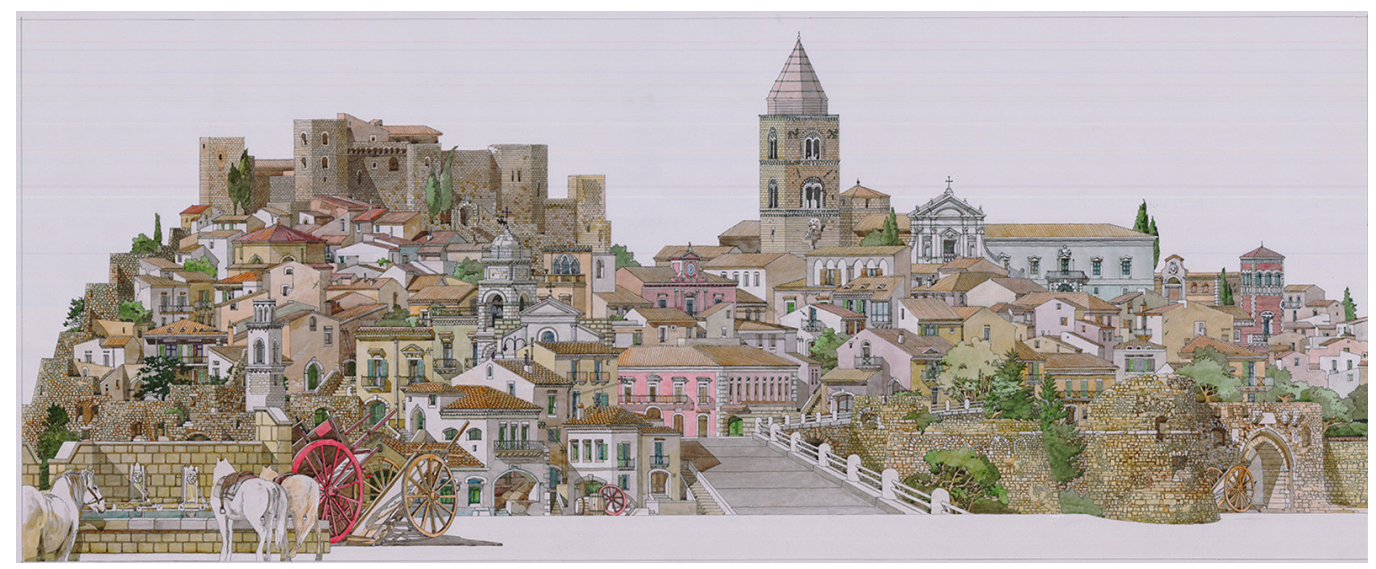




\section{II motivo di una scelta}

"Disegno sempre anche quando scrivo": così afferma Günter Grass [2], ed aggiunge che "lo scrivere e il disegnare sono correlati". Queste riflessioni mi hanno dato modo di pensare che, nel caso di Michele Cascarano, potrebbe essere valida la parafrasi "Disegno sempre anche quando penso", con cui abbiamo titolato questo contributo.

È difficile presentare in uno spazio ridotto esperienza, attività creativa e tecnica di un architetto, e la sua poetica: non è facile soprattutto andare al di là della semplice descrizione delle opere per tratteggiare quello che possiamo definire anche un "disegno dell'anima" [3]. Esso pervade tutta la sua produzione, riproduce nel lavoro i temi e le questioni affrontate ma al tempo stesso indaga le suggestioni e le tante cose che incontra, che meritano attenzione fino ad invadere totalmente spazi e superfici di tutta la sua casa-studio sulla quale torneremo.

Egli vive disegnando e disegna vivendo luoghi e architetture. II nostro interesse è motivato quindi dal fatto che indagare su questa figura silenziosa ci consenta di aprire uno spaccato su alcuni aspetti della storia della professione di architetto nel secondo Novecento; al tempo stesso offra lo spunto sia per dibattere sul senso che questa professione può avere nel XXI secolo, sia per interrogarci su quale valore e utilità possono avere, nella formazione, i linguaggi della rappresentazione tradizionali come il disegno, le tecniche pittoriche, i plastici e le maquette.

La sensibilità dell'occhio che cattura e l'operosità e la maestria della mano che traduce in segni, campiture o in ritagli per la costruzione di modelli possono essere ancora obiettivi sui quali lavorare con i giovani che si avvicinano a questa professione?

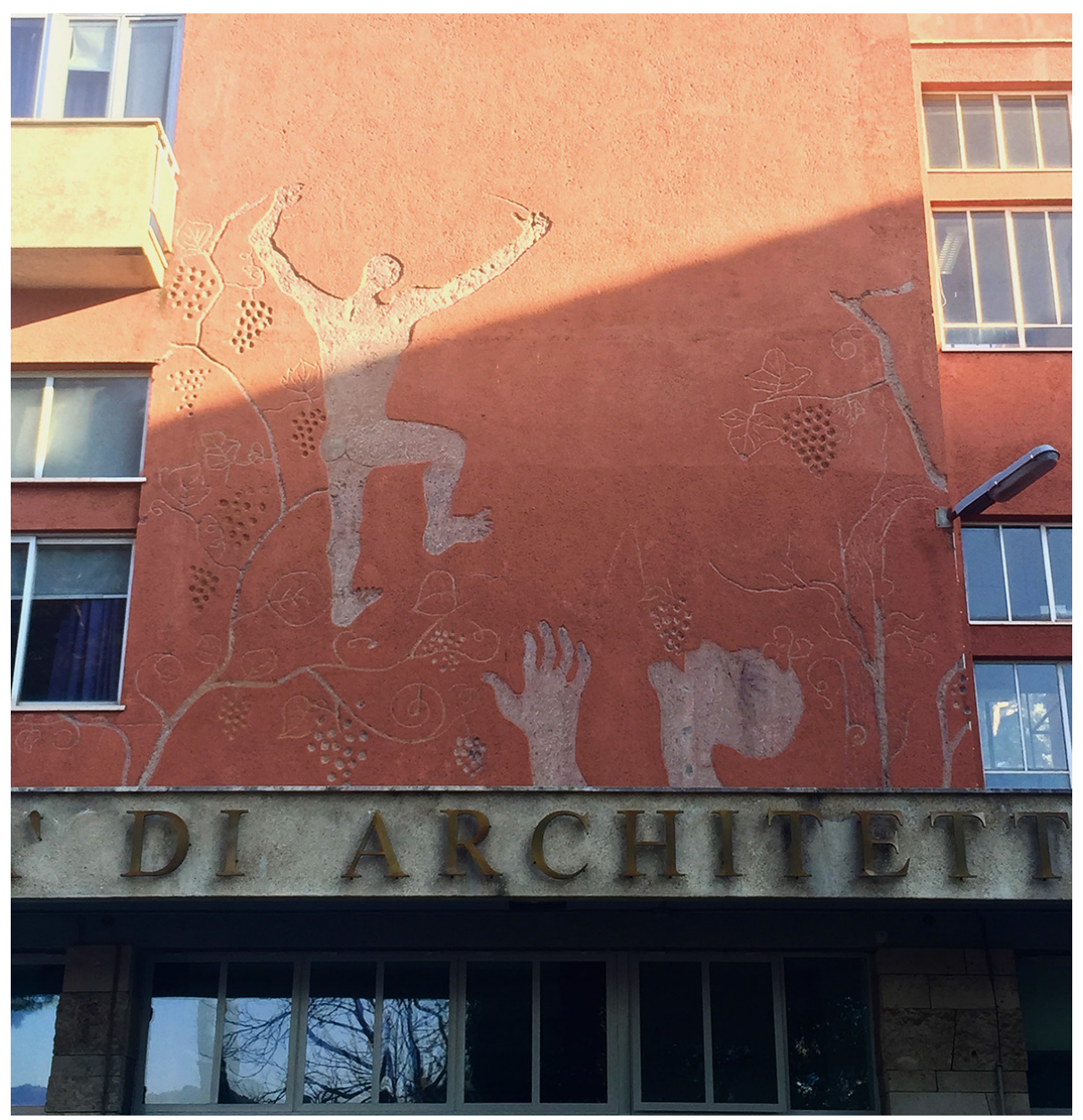




\section{Cenni biografici: la formazione nel contesto della Scuola di Architettura romana del secondo Novecento}

Il percorso formativo di Michele Cascarano [4] ci permette di tratteggiare uno spaccato sulla complessità culturale romana relativa all'Architettura negli anni Sessanta. Nel suo percorso incontra docenti come Gianluigi Nervi con i suoi assistenti Antonio Michetti [5] e Sergio Musmeci [6], e architetti che hanno scritto la storia dell'architettura del Novecento: Enrico del Debbio [7], allora Direttore dell'Istituto di Disegno e Rilievo dei Monumenti della Facoltà di Architettura (fig. I), e Leonardo Benevolo, giovanissimo titolare della cattedra di Storia e Stili dell'Architettura.

Deve essere stato l'incontro con il Disegno a condizionare il suo percorso e di conseguenza il suo futuro professionale ed espressivo. In particolare gli insegnamenti di architetti, allora giovani assistenti Gaspare De Fiore [8] e Angelo Marinucci [9], che teneva corsi extrauniversitari di acquerello che Cascarano seguiva con altri compagni di università, o ancora di Luigi Vagnetti [I0] che nel corso di Disegno dal Vero allenava i giovani studenti al Disegno "sia come mezzo di espressione, sia come strumento di lavoro, sia infine come efficacissimo aiuto nello studio delle altre discipline artistiche" [Vagnetti 1955, p. 5]. Nel testo didattico sul quale certamente anche Cascarano ha studiato, Vagnetti afferma: "E quello del Disegno dal Vero l'unico modo di esercitare contemporaneamente la mano, il cervello ed il cuore [...]; è il modo più efficace per considerare contemporaneamente la opportunità evidente delle analisi e la necessità assoluta della sintesi; è l'unico modo infine per raggiungere la esplicazione di una personalità completa ed indipendente, passando attraverso agli stadi transitori di indagine obiettiva; che permettono poi la massima libertà di espressione soggettiva, non subordinata ad alcuna convenzione ma solo cosciente della realtà vera delle cose" [Vagnetti 1955, pp. 8, 9].

È evidente in queste parole la rivendicazione netta del Disegno come modo per conoscere e impadronirsi della realtà per poi rappresentarla in modo soggettivo con libertà creativa: tanto che "i Pittori, gli Scultori e gli Architetti" sono comunemente definiti "Maestri del Disegno" [Vagnetti 1955, p. 5].

Forte certamente di questi insegnamenti, dopo la laurea entra nello studio Valle [I I] dove lavora tutta la vita come Art Director, al tempo stesso non abbandona il mondo universitario, svolgendo attività didattica [12].

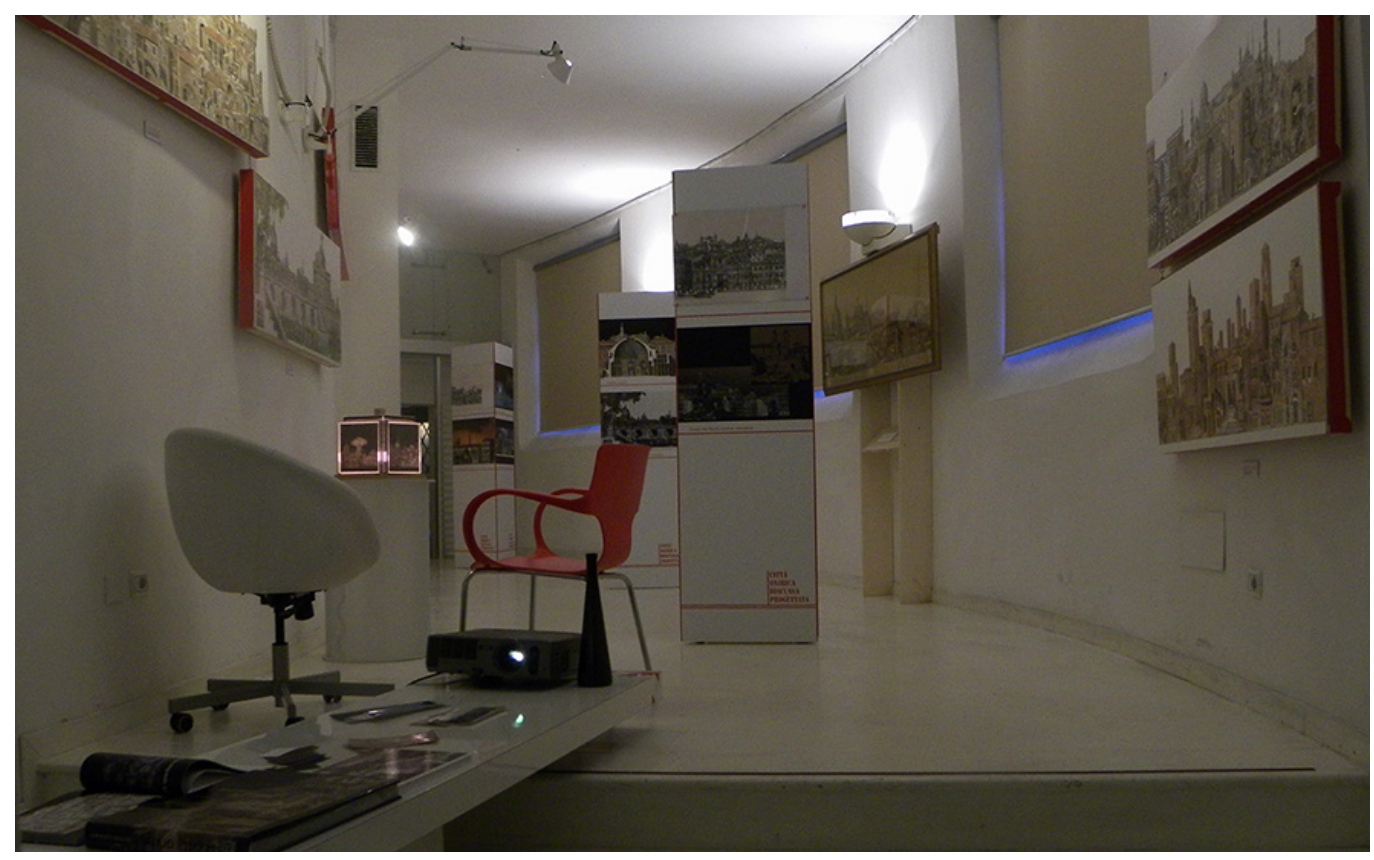


Per definire Cascarano ci sembra quindi appropriata la definizione di "Maestro del Disegno": lo è per la sua attività didattica, lo è all'interno dell'attività professionale, dove elabora complesse e dettagliatissime prospettive di progetto o minuziosi plastici, lo è perseguendo con passione e accanimento il suo naturale bisogno di rappresentare la sua idea di città, che si svincola dalla riproduzione fedele del Disegno dal Vero per spaginarsi in narrazioni dove le quinte urbane si aprono e le facciate degli edifici diventano una narrazione continua, in un racconto che si fa percorso dinamico dell'osservatore.

La sua lunghissima collaborazione con lo studio Valle, molto attivo in concorsi e progettazioni internazionali, gli offre numerose opportunità di girare il mondo. Acquisisce quindi una particolare capacità di afferrare il genius loci e di tradurlo graficamente.

\section{Le mostre}

L'artista a partire dal 1965 partecipa a esposizioni in Italia [ I 3] e all'estero [ 4 ] riscuotendo interesse e apprezzamento.

Alcuni titoli delle esposizioni rivelano al visitatore il portato poetico: in "La città onirica" [I 5], ad esempio, citando Calvino si sottolinea che "è l'umore di chi la guarda che dà alla città la sua forma" [ 16] per introdurre "sette grandi opere ad acquerello in cui fantasie urbanistiche, vedute irreali di architetture reali, profili di città visti attraverso un sogno, ricordano diari di viaggio" [I7]; "La stanza dei sogni” anticipa le sensazioni comunicate dalle opere esposte, mentre "Mediterraneo" [ I 8] suggerisce un ambito culturale-geografico di riferimento.

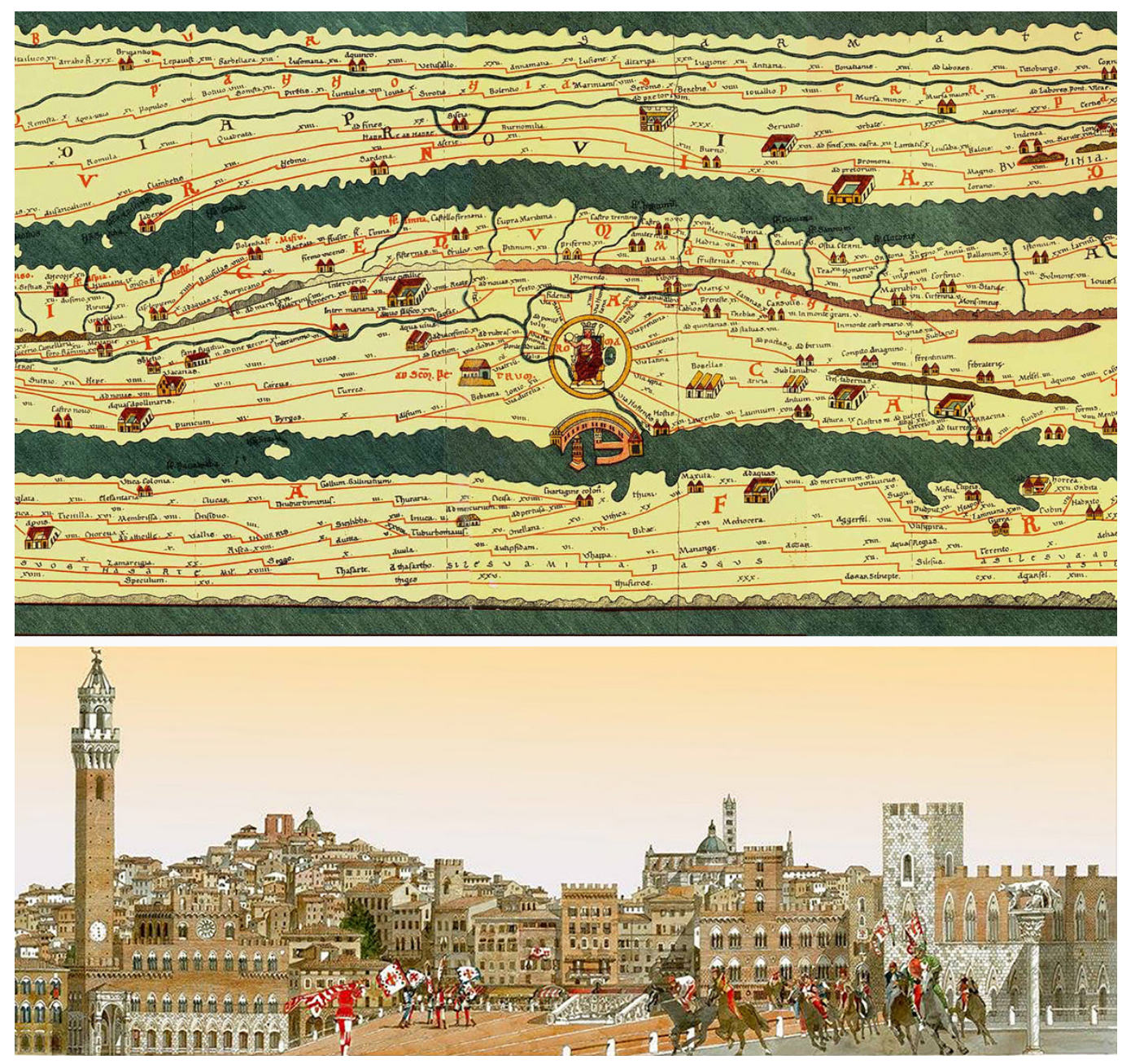


Diversamente da queste iniziative, focalizzate su temi specifici, la mostra "città onirica, città discussa, città progettata" alla Casa dell'Architettura di Roma [19] propone una riflessione sulla produzione ampia e variegata di Cascarano, consentendo una conoscenza della personalità sfaccettata dell'architetto (fig. 2). Sono infatti esposti i grandi acquerelli (anche con varianti cromatiche, viraggi in seppia $\mathrm{O}$ in bianco/nero) sulla città di Roma memorizzata ma anche modelli di progetto e di lampade.

Fig. 4. In alto, piazza S. Marco verso la Basilica, Venezia, Antonio Canal detto il Canaletto, disegno a penna; in basso

piazza s. Marco,Venezia, acquerello, Michele Cascarano (montaggio grafico I. Passamani).

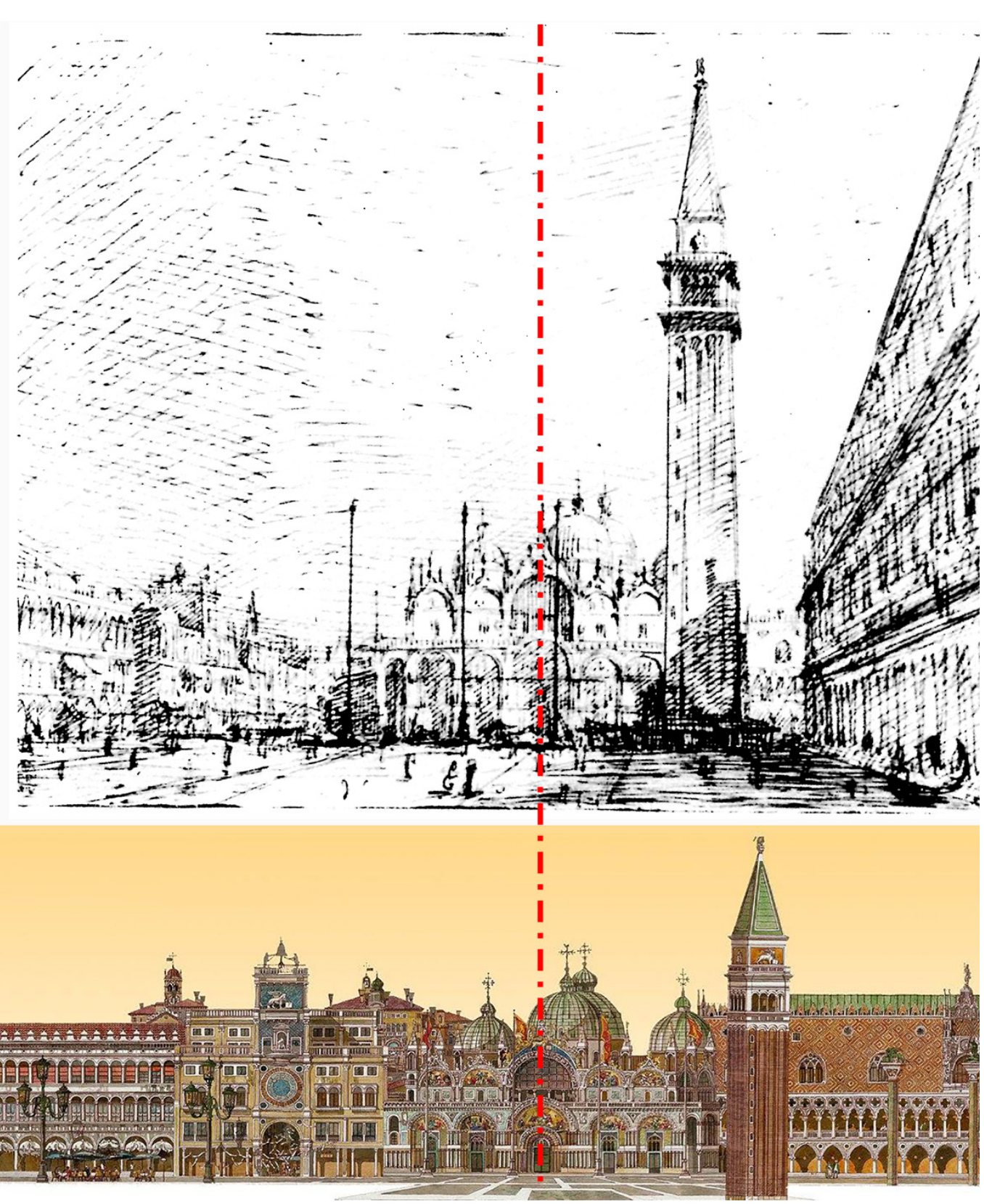

I precedenti, le suggestioni, i riferimenti

La produzione grafica di Cascarano permette di individuare alcuni riferimenti celebri, che qui accenniamo con parole chiave e sintetiche considerazioni, riservando a studi futuri ulteriori approfondimenti. 


\section{I disegni di viaggio o per il viaggio}

I grandi acquerelli in cui sono srotolati in successione i fronti urbani di città italiane e straniere richiamano la Tabula Peutingeriana [20] per la similitudine di montare in sequenza un racconto di luoghi; nel caso dell'antica mappa i luoghi sono descritti a scala territoriale ed il racconto e l'uso sono funzionali al viaggio, le raffigurazioni di città del Nostro sono invece narrazioni ex post. Entrambe costringono chi ne fruisce a compiere un viaggio virtuale e mentale nei luoghi che il disegno rappresenta (fig. 3).

Inevitabile, poi, l'accostamento con i disegni prodotti dai viaggiatori-disegnatori protagonisti di diversi Grand Tour nel XVIII e XIX secolo, che durante i viaggi lungo l'Italia ne subivano la fascinazione: hanno lasciato panoramiche ad ampio raggio in cui l'intento era fissare tutto il visibile.

Sicuramente anche Cascarano, viaggiatore per lavoro e per passione, non vuole tralasciare nulla: possiamo riconoscere le sue città e le piazze dall'apparente fedeltà con cui sono disegnate... ma ad una osservazione attenta emerge quella libertà di espressione soggettiva (evocata da Vagnetti) che trasforma paesaggi a memoria in onirici: stupisce, quindi, riconoscere immediatamente una piazza e poi scoprire che non è esattamente così... uno stupore salutare, ci fa avviare un processo critico (fig. 4), ci fa sentire architetti!

\section{I vedutisti}

Si ravvisano, nell'impostazione dei punti di vista delle sue visioni prospettiche, i riferimenti alle raffigurazioni dei vedutisti: il Canaletto e Francesco Guardi per le visioni veneziane (fig. 5), Filippo Juvara e Gianbattista Piranesi per le vedute urbane di Roma (fig. 6). Alcune di esse, che qui proponiamo in connessione con quelle di Cascarano, sono tratte dal testo di Vagnetti

Fig. 5. In alto, veduta della Riva degli Schiavoni,Venezia, Francesco Guardi, inchiostro e acquerello; in basso veduta verso piazza S. Marco, Venezia, acquerello, Michele Cascarano (montaggio grafico I. Passamani).
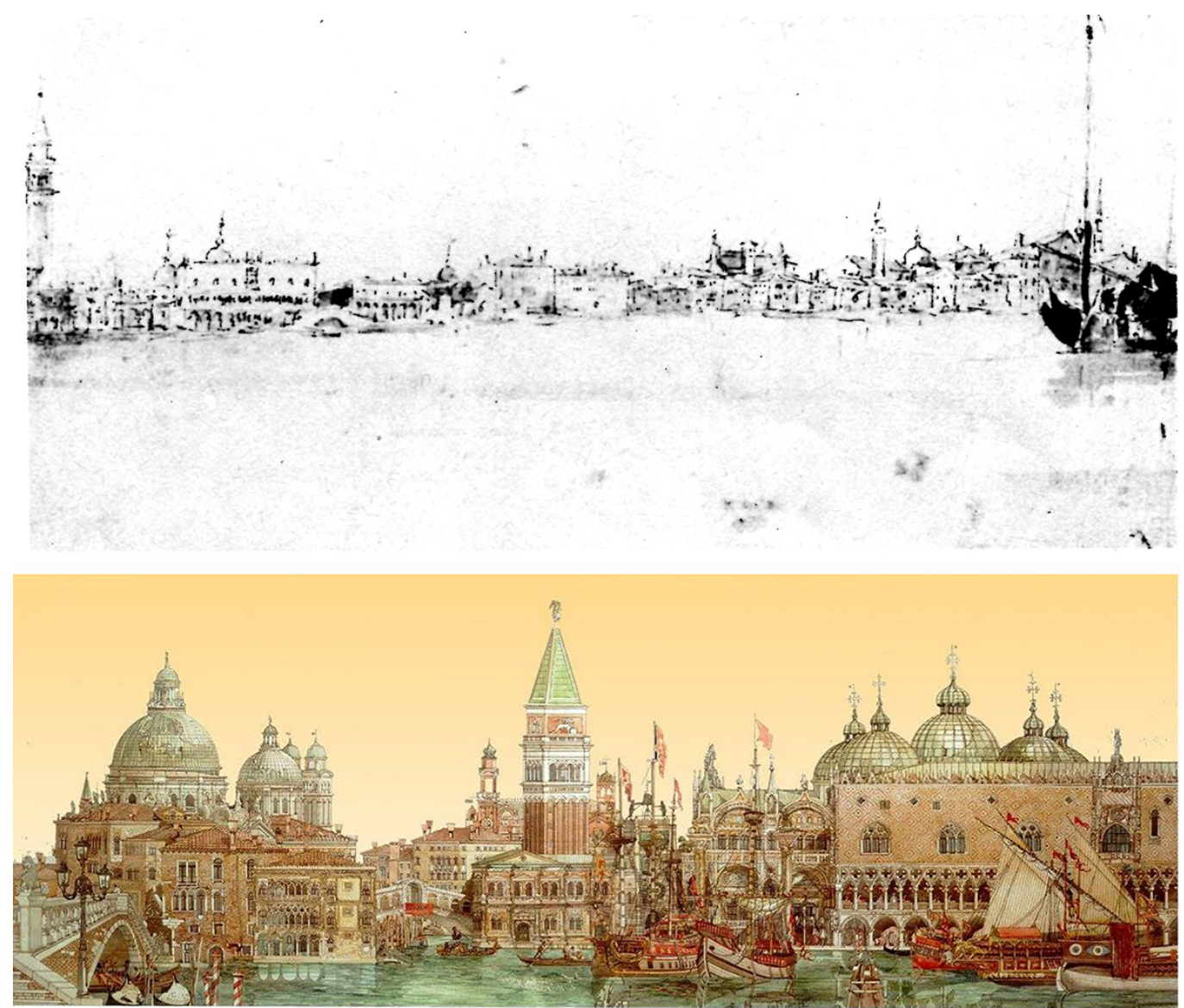
Fig. 6. In alto, veduta del Campidoglio da piazza Aracoeli, Roma, Filippo Juvara, penna e acquerello; in basso veduta de Campidoglio da piazza Aracoeli, Roma, acquerello, Michele Cascarano (montaggio grafico Passamani).
Fig. 7. In alto, Fontana di Trevi, Roma, Giambattista Piranesi, incisione; al centro Fontana di Trevi, degrado, Roma, Gaspare de Fiore, disegno.

In basso, l'interpretazione di Fontana diTrevi all'acquerello, Michele $\mathrm{Ca}$ scarano. La rielaborazion grafica (I. Passamani)

assume come riferiment l'asse di simmetria dell fontana e quello della facciata della chiesa de SS. Vincenzo e Anastasio a Trevi. Tale facciata costituisce il dato comune tra Piranesi e Cascarano.
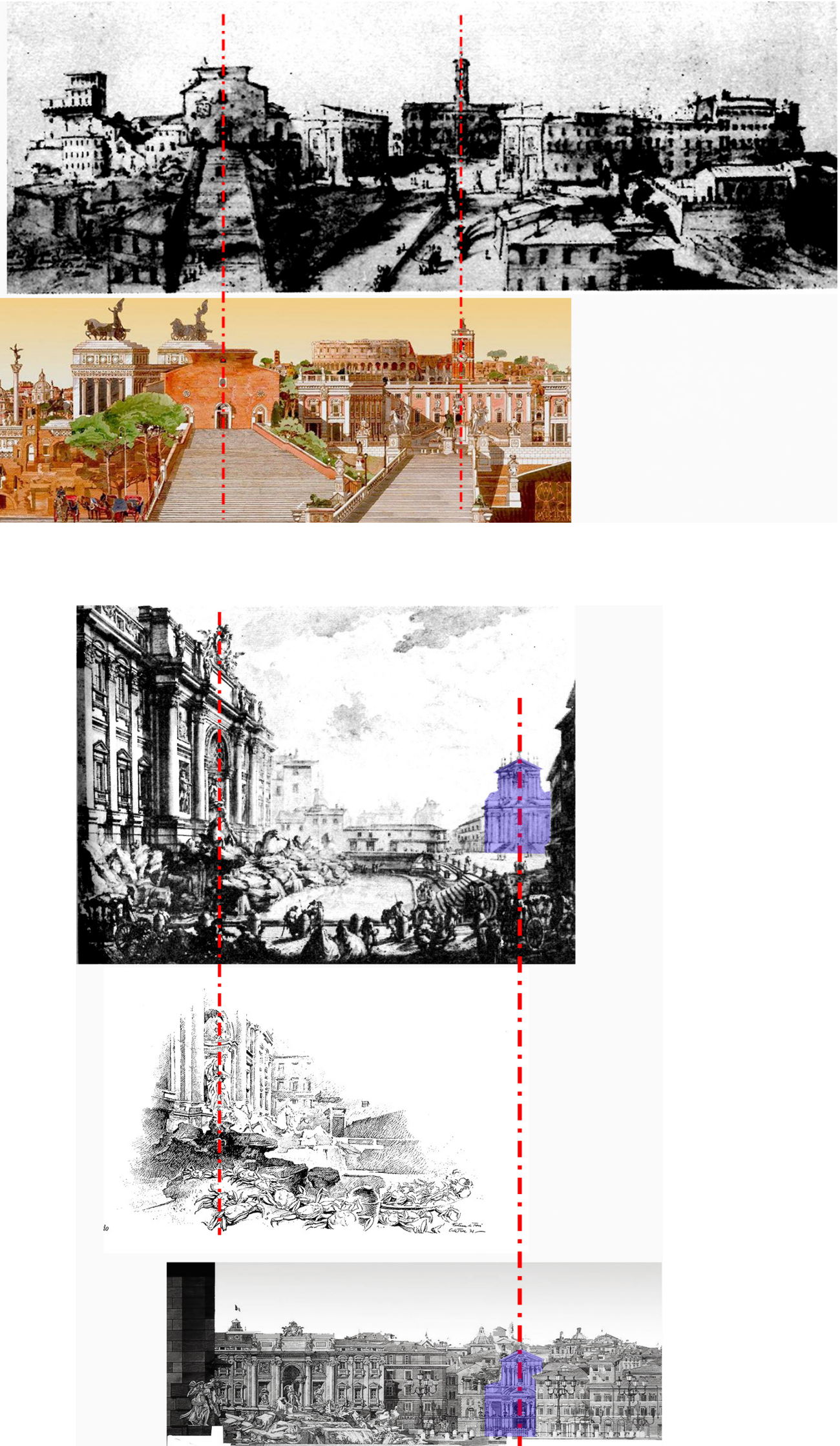
[Vagnetti 1955]: evocazioni e suggestioni, punti di vista che sicuramente il Nostro ha introiettato negli anni della sua formazione.

Illuminante il montaggio diacronico di diverse interpretazioni della Fontana di Trevi: una sequenza temporale che parte dalla vista angolata di Piranesi, passa per un disegno di Gaspare de Fiore fedele al taglio prospettico, arriva a Cascarano che apre la scatola urbana ponendo la fontana sullo stesso piano di quadro della chiesa dei SS. Vincenzo e Anastasio (fig. 7).

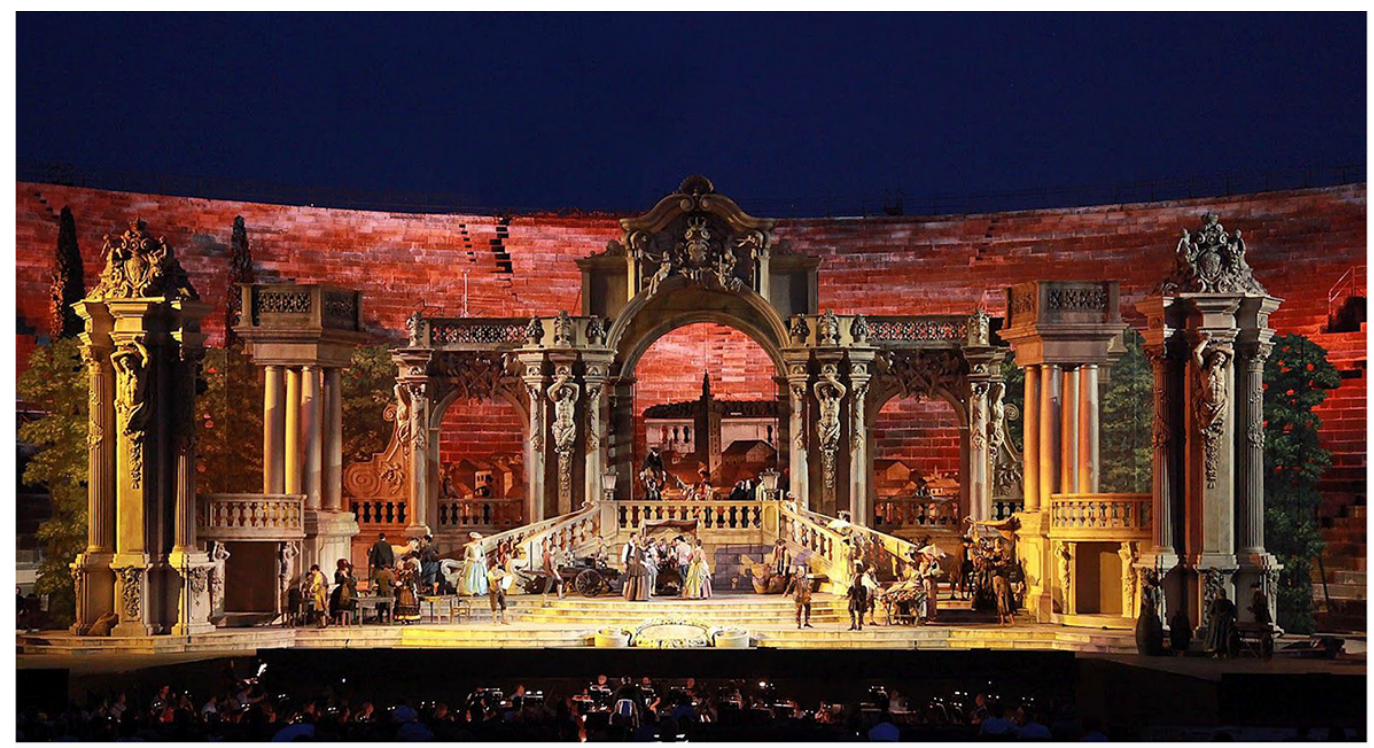

Fig. 8. La realizzazione de teatrini, piccole macchine sceniche in cartoncino dipinto, riguarda ad esempio citazioni celebri come quella della scena del $2^{\circ}$ atto del Don Giovanni di Mozart. In alto un'immagine (Arena diVerona, regista Franco Zeffirelli), regista Franco Zeffirelli), Don Giovanni di Mozart, Don Giovanni di Mozart cartoncino acquerellato, taggio grafico I. Passamani)

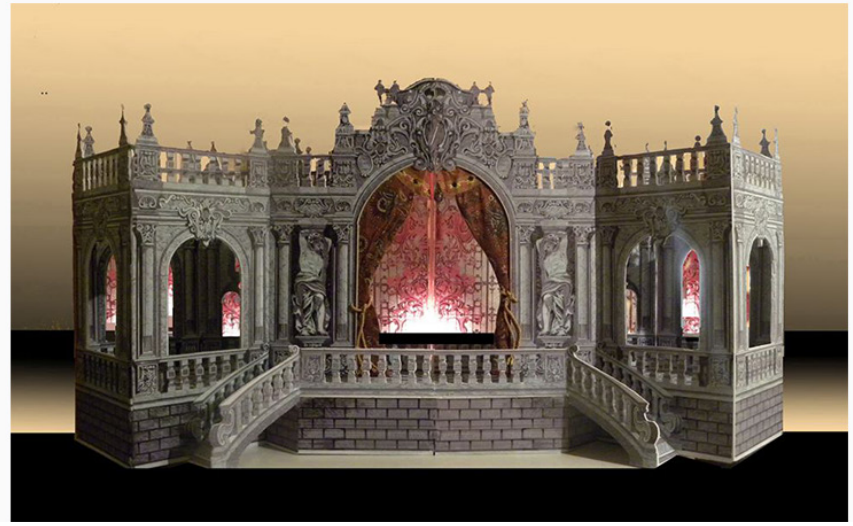

\section{Lo spazio onirico}

II suo spazio privato è onirico: come specchio di sè, come suo prolungamento, esso raccoglie le sue interpretazioni grafiche della realtà esterna accanto a oggetti concreti del mondo esterno e a costruzioni oniriche, come i Teatrini di Viaggio (fig. 8).

È uno spazio antropomorfico, connotato da quell'horror vacui che caratterizzava le antiche Wunderkammern: stanze delle meraviglia che raccoglievano, per la gioia del collezionista di "messa in mostra" [Basso Peressut 1997, pag. IX], gli oggetti raccolti. Quelle mirabilia che rappresentano l'universo interiore di Cascarano (fig. 9). 


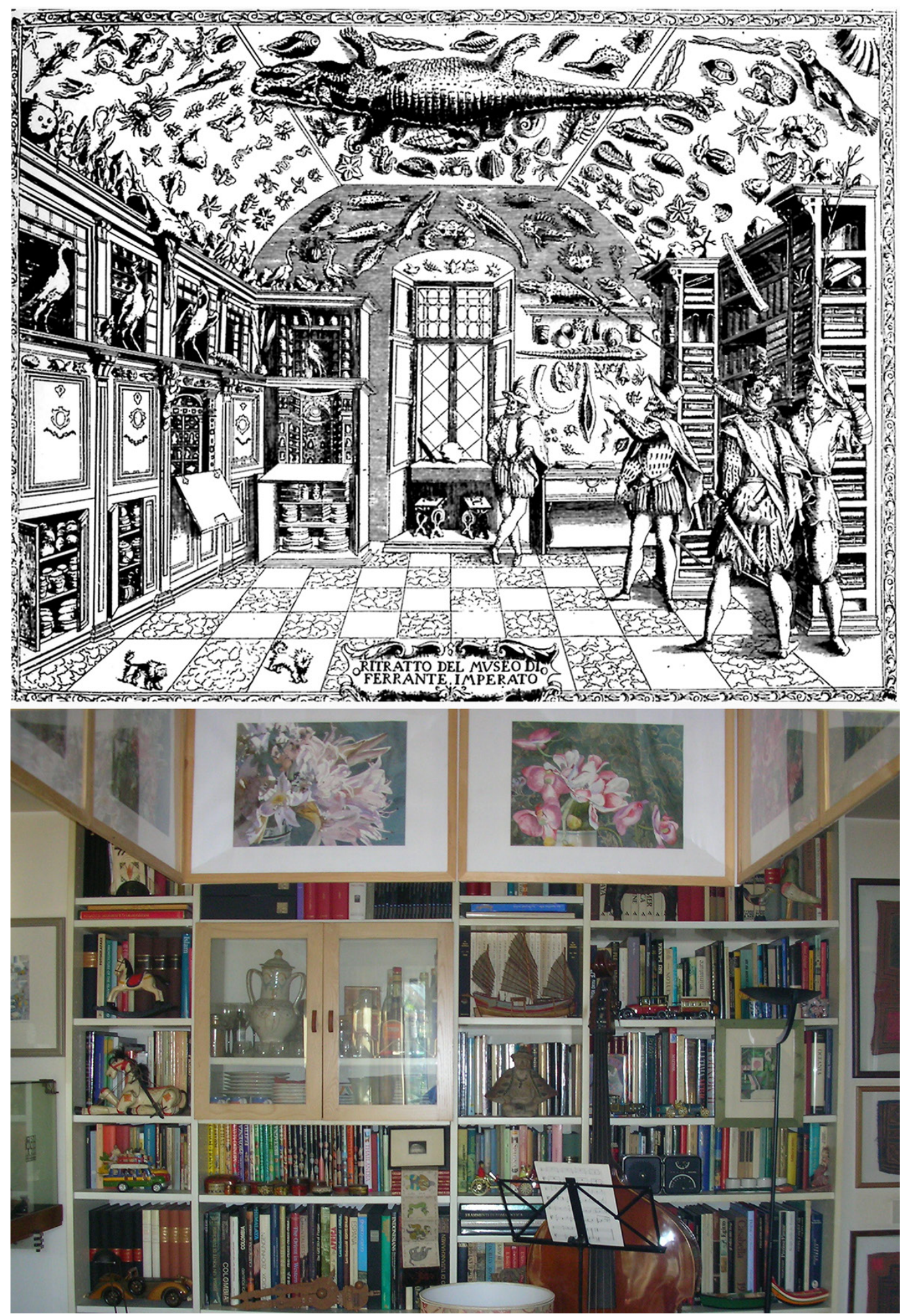




\section{Questi artifici di luoghi e città sono una narrazione architettonica. Con quale metodo critico smontare e rimontare questi disegni di architetture senza tempo?}

Le riflessioni raccolte in questo contributo costituiscono una forma di sintesi dell'esperienza che, per una fortunata coincidenza, si aggiunge ad un giudizio personale e profondo della figura di uno dei tanti maestri minori che riempiono la storia reale della nostra presenza sulla Terra.

La nostra complessa cultura dell'abitare e vivere lo spazio che immaginiamo, che pensiamo, che progettiamo, che costruiamo, rappresenta un continuo disvelamento di intrecci profondi tra ideazione e pensiero, immaginazione e costruzione dell'architettura disegnata con quella che è stata edificata.

Si tratta di pagine inedite che, proiettate nel dibattito contemporaneo sull'architettura del secondo Novecento e del nuovo millennio, illuminano con una luce più chiara il senso dell'abitare contemporaneo in uno specifico contributo che immette, nell'apparato storico-critico e documentario della Comunità, traiettorie di ricerca di dimensioni inaspettate, come le opere di moltissimi maestri che non conosciamo affatto [2I].

È mio interesse tentare di evidenziare caratteri e questioni metodologiche dell'intreccio fittissimo tra fondamenti teorici e la ricerca stessa in architettura, attraverso la rappresentazione ed i disegni di Cascarano.

Una seconda parte, in forma personale, intende definire una reale immersione nelle centinaia di tavole, disegni, vere incisioni, acquerelli, che fissano sulla carta il territorio quasi infinito di città visitate e immaginate, tra memoria e oblio di architetture senza tempo.

II suo lavoro rappresenta e mostra l'esistenza di una ricerca individuale, ad un tempo intesa come opera collettiva dell'essere architetto che ripone nell'arte del disegno una visione ed una originale esperienza della realtà.

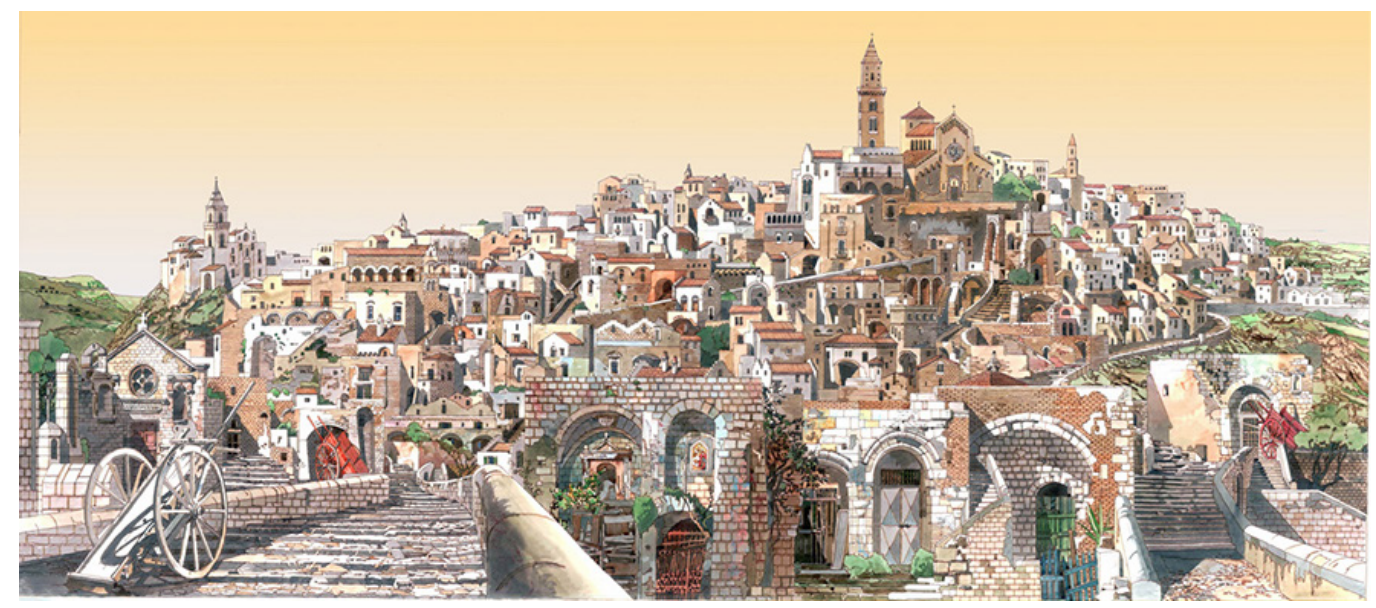

Da questo fondamento egli trae l'energia e la forza rappresentativa di disegnare architetture tendenziosamente collocate tra rilievo, memoria e progetto.

La questione che emerge è una sorta di assunto, di vocazione a considerare la città da un punto di vista analitico che è un momento fondamentale per lo sviluppo della conoscenza e del progetto nel campo, se pur parziale, del fare architettura.

II rapporto tra architettura, razionalità ed invenzione rappresenta la realtà costruita in modo molto concreto e molti disegni di invenzione, di parti di città analoghe composte con parti ed elementi di altri luoghi, sono un corpus disciplinare unico, senza il quale qualsiasi avanzamento disciplinare sarebbe parziale: è nostra responsabilità farlo conoscere e vivere nel tempo della storia. Questi assunti teorici e critici significano spesso per noi la fede, più o meno cosciente, nel Disegno come pratica professionale e sperimentazione didattica che rappresenta sempre una sorta di scorciatoia verso il progetto e la costruzione. 


\section{La riduzione della realtà dell'architettura a complesse visioni e narrazioni}

Questa riduzione della realtà dell'architettura a complesse rappresentazioni che sono visioni e narrazioni di processi di sintesi, non impedisce di sviluppare forme diverse di conoscenza e interpretazione dei luoghi della storia, anche solo immaginati come costruzione analoga di città.

Ponendoci innanzi a questi disegni scorgiamo, indipendentemente dalla dimensione, da parti ed elementi, aree e luoghi, i caratteri di fatti urbani la cui presenza costituisce proprio l'anima critica che riempie la vita di Michele con il Disegno come Angelo necessario. Ha visto Roma, Venezia, il Cairo, Barcellona (fig. 10) innumerevoli città e, con narrazione ossessiva, traccia geografie autentiche, identifica la natura dei paesaggi, individua configurazioni possibili anche a costo di visualizzare cose immaginate, trasposte in nuove composizioni urbane.

Queste tavole lunghissime di città, in qualche modo ricomposte in una fissità onirica, sono una trama fitta e continua di monumenti e architetture stilisticamente rilevanti, che si pongono come speranza visionaria di una bellezza reale. Credo abbia vissuto questa parte della sua vita disegnando, nel tentativo ossessivo di procedere alla costruzione di una 'città sperata', di tante città e di servirsi di una serie di elementi diversi, collegati tra loro ma il cui significato è proprio nell'esperienza di definirne una possibile 'forma ideale'.

La città dunque per sua natura non è una creazione unica e può essere ricondotta dal pensiero a idee di base, a sintesi di una formazione straordinaria. Questi disegni si pongono come un lavoro compiuto di ricollocazione in una fissità eterna di quel momento, di quella osservazione svolta in più direzioni, dalla ricerca reale alla bellezza. L'individualità delle parti e dei singoli elementi rappresentano indagini dirette, suoi discorsi e ragionamenti di esistenza che dal procedimento classificatorio raccolgono più profonde poetiche personali.

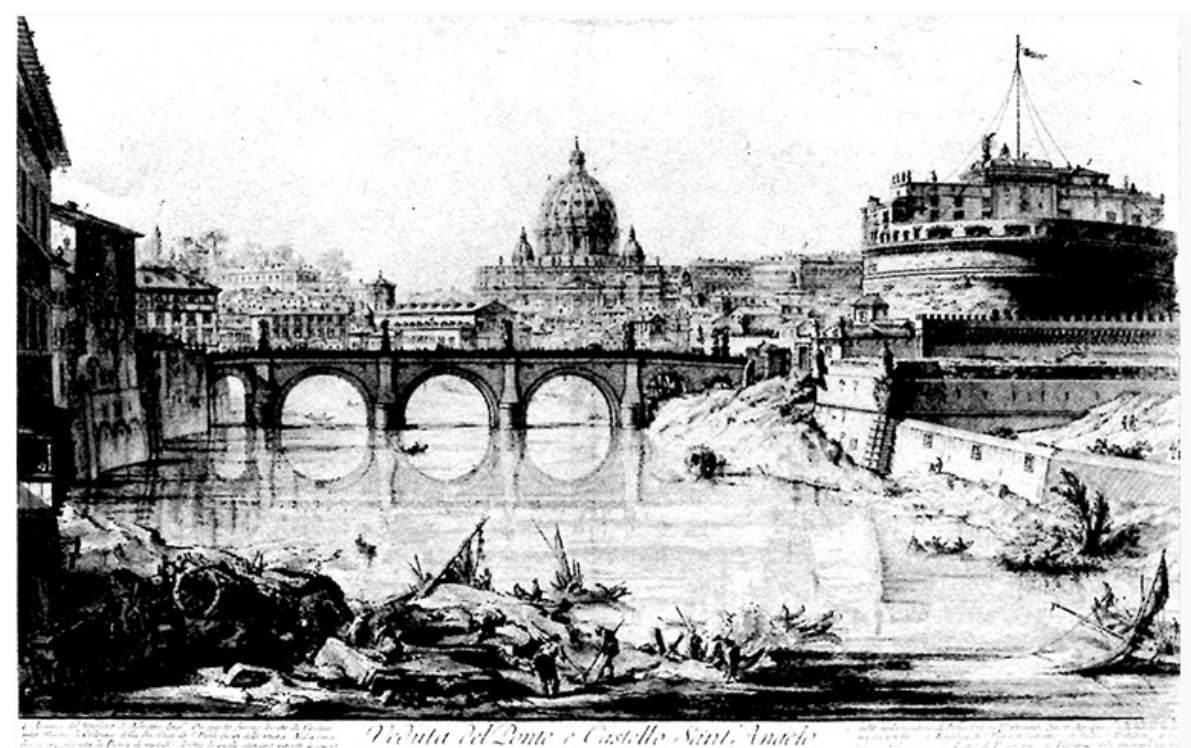

Fig. I I. In alto, Veduta del Ponte e Castello Sant'Angelo, Gianbattista Piranesi; in basso veduta di Castel Sant'Angelo, Roma, acquerello, Michele Cascarano (montagrio grafico I. Passamani).

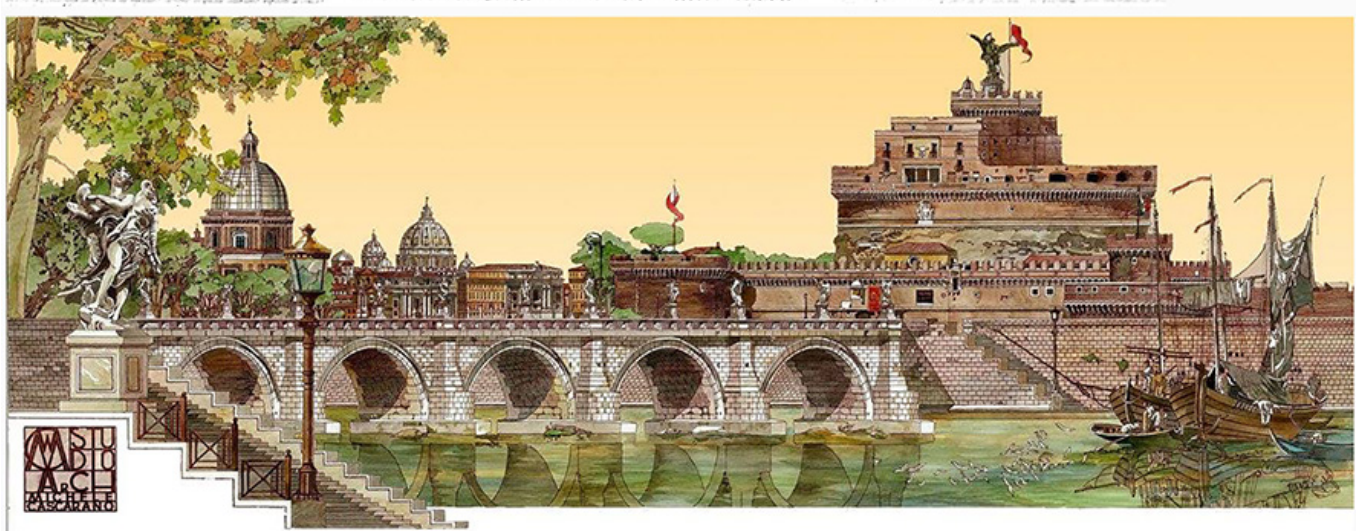


Questa forma di lavoro, di indagine scientifica sulle città e sui loro caratteri tipo-morfologici, si presenta come visione di approcci metodologici tesi a ritrovare il progresso nella storia e come riconoscimento dell'autonomia dell'architettura dai fatti umani e da quelli fenomenologici.

In questo procedere critico della conoscenza ho cercato di precisare un avvicinamento al problema del progetto in architettura che sembra sottendere ai disegni intensamente legati a intrecci di visioni radicate nella memoria, nel sogno, nell'immaginario e nell'illusione di una realtà diversa, teorica.

Credo non sia necessario comprendere le tecniche del disegno ma cogliere quell'intreccio dialettico tra realtà urbana osservata e gli elementi dell'architettura tracciati nella precisione del dettaglio, come significato profondo di ogni esperienza di progettazione e di costruzione. In tutti i disegni, da quelli a tratto ai finissimi acquerelli, è evidente una maestria nell'uso degli strumenti propri di un corpus disciplinare che si pone come obiettivo la costruzione e l'avanzamento dell'architettura come patrimonio di conoscenze e di tensioni critiche. Essi rappresentano le individualità, i fatti urbani anche in una forma ideale che potremmo porre senza tempo tra i disegni di Canaletto e Piranesi (fig. I I).

D'altra parte l'analisi ed il progetto si applicano alla città costruita, alle sue architetture e con queste è possibile confrontarsi, fare scelte formali e teoriche.

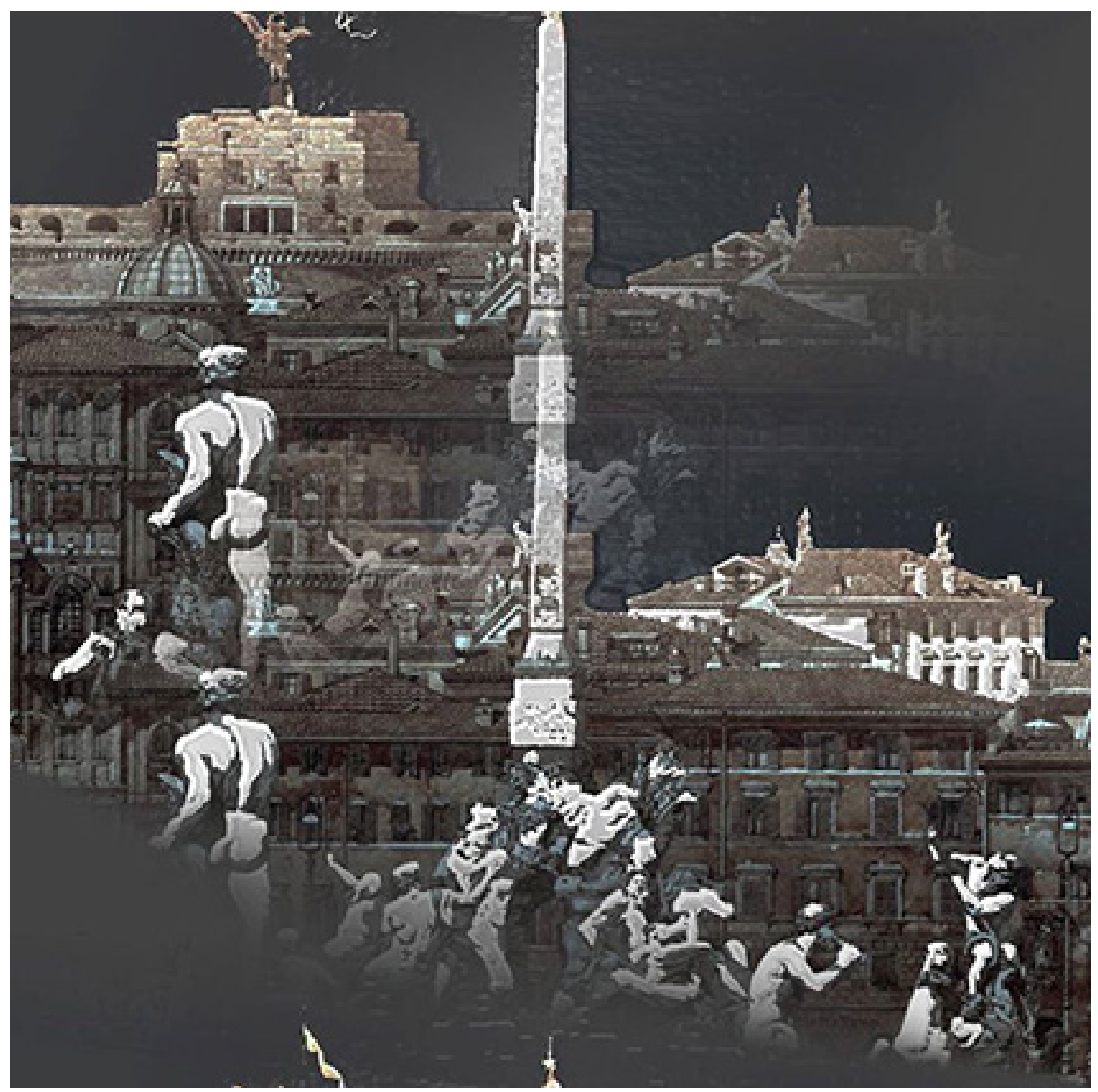


In queste opere si riconosce questo carattere che lega ogni pensiero al disegno, alla ricerca tesa a rendere chiara la natura dell'architettura e delle città in una visione di possibili trasformazioni e valori civili che investono quotidianamente il lavoro di un architetto, dei fallimenti, della precarietà, delle fragilità delle azioni e di poter cambiare le cose attraverso l'architettura.

Le mie considerazioni riguardano quindi un discorso espresso in forma razionale che sottende l'adesione ad una 'visione del mondo' che investe il problema del significato dell'architettura come senso etico e civile dell'umanità. Questo discorso critico ha origine sul ruolo teorico di queste esperienze per tentare di ri-costruire un possibile discorso scientifico sull'architettura, a partire dalla quantità di opere e disegni che attraversano le vite di maestri minori, spesso sconosciuti che si presentano a noi.

\section{Conclusioni. Una mostra per dibattere e riflettere sul senso e ruolo del disegno di architettura come conoscenza e progetto della realtà}

Da sempre, dal primo incontro, è mio convincimento che la conoscenza di Michele Cascarano rappresenti un'occasione culturale e formativa importante per la giovane scuola di Architettura di Matera: presentare criticamente il lavoro di questo grande disegnatore, ostinato e incessante, di una vita dedicata al progetto, può colmare alcune assenze nella storia della rappresentazione e del Disegno come Conoscenza. Questa attività critica, che si avvia a molte precisazioni per la definizione di una mostra dei suoi lavori, mi riempie di orgoglio e di senso di responsabilità, con un filo di preoccupazione per la dimensione numerica delle opere e per la loro indiscussa e altissima pregevole qualità e ricchezza di contenuti.

II ruolo svolto dalla conoscenza dell'architettura, di una sua pratica condotta per tutta la vita, tratteggia una prassi costante di chi osserva modificando continuamente l'ambiente costruito, edificando nei disegni in modo alternativo una sorta di 'città sperata', trasformando anche le rovine in luoghi e cataloghi perfettamente compiuti riempiti di una seconda natura di esistenza e di bellezza.

Tra gli artifici di questa straordinaria stabilità delle forme delle cose edificate dall'uomo, della loro caducità, Michele opera ragionevolmente delle scelte e si riserva di consegnarle ad una maggiore durata possibile, offrendo a sé stesso e al mondo luminose rappresentazioni del senso stesso del fabbricare: lo fa istituendo una visione stabile delle città, delle architetture che ricompongono la 'scena fissa' della permanenza dell'arte nella storia e nel tempo.

Così l'atto del fabbricare e l'opera d'arte della creazione architettonica risultano per sempre saldati e collegati per ritrovare e configurare un mondo abitabile non soggetto alle rovine del tempo e all'incuria degli uomini.

L'osservatore viene coinvolto nell'avventura vertiginosa di un pensiero, il cui atto del costruire rappresentato da questi mosaici di città (fig. 12) è il nesso, il senso di esistenza, del "pensare disegnando per comprendere alcune realtà del mondo".

\section{Note}

[I] L'attività di studio sulla figura di Michele Cascarano è stata avviata da entrambi gli autori del presente contributo, che condividono l'interesse ad approfondire i tanti aspetti della produzione progettuale e artistica dell'architetto. Si segnala che Ivana Passamani è autore dei primi sette paragrafi e Antonio Conte dei tre paragrafi finali.

[2] Günter Grass (1927-20 I5), scrittore e poeta, scultore e pittore, ha ricevuto il Premio Nobel per la letteratura nel 1999.

[3] Ci piace prendere a prestito questa definizione citando il titolo di un testo di Elio Vigna, 200 I.

[4] Nasce a Melfi (Potenza) il I 0 febbraio 1936. Dopo la fine della guerra la sua famiglia si trasferisce a Roma, città nella quale tuttora vive e lavora. Qui segue il liceo, iscrivendosi nel 1954 alla Facoltà di Architettura dell'Università degli Studi “La Sapienza”. Si laurea a pieni voti nel 1961.

[5] Chi, come me, ha avuto la fortuna di conoscere l'ing. Antonio Michetti (1927-20 I0) e di seguire le sue lezioni di "Tecnica delle Costruzioni", concorda sul fatto che egli fosse "il più amato degli ingegneri romani". Non stupisce quindi il conferimento della Laurea Honoris Causa in Architettura (La Sapienza, Roma, 03/03/2003): egli era profondamente architetto, come dimostra la sua affermazione "La prima progettazione non è statica, ma architettonica". Nella sua lunga attività professionale Antonio 
Michetti ha sempre operato coerentemente con la lezione del suo maestro Pier Luigi Nervi, contribuendo alla definizione dell'immagine dell'architettura italiana contemporanea.

[6] Sergio Musmeci (1926-1981), ingegnere strutturista, inizia la sua attività con Morandi e Nervi; suo il progetto strutturale dello Stadio del Nuoto al Foro Italico di Roma (1959), il cui progetto architettonico è firmato da Del Debbio e Vitellozzi.

[7] Enrico del Debbio ( 189 | - 1973) firma il progetto della Facoltà di Architettura di Valle Giulia a Roma.

[8] Gaspare de Fiore ( I926-20 I I), architetto e docente, negli anni '50 illustratore con Jacovitti e altri della testata "IIVittorioso".

[9] Angelo Marinucci (1909-1994) è architetto, docente e pittore. Libero docente alla Sapienza, collabora con Vagnetti al corso di Disegno dal Vero.

[10] Luigi Vagnetti (1915-1980), teorico dell'architettura e del Disegno, docente universitario e progettista, dà un contributo scientifico fondamentale anche nel campo del rilievo architettonico, che considera collegato in modo imprescindibile alla rappresentazione della realtà.

[ I I] Cesare Valle (1902-2000), architetto, è docente di urbanistica alla facoltà di Ingegneria della Sapienza; è autore di numerosi progetti architettonici e di piani regolatori.

[12] Insegna nei corsi di Disegno dal Vero, Tecnica dell'acquerello e Cromatismo in architettura.

[1 3] Roma, Ovindoli, Rocca di Mezzo, Assisi, Civitavecchia.

[14] Tokio, Cipro.

[ I 5] Mostra alla Temple Gallery, Roma 29/9-15/I0 1998.

[16] È la città di Zemrude. Italo Calvino, 2019, Le città e gli occhi.

[17] In Roma C'è - Arte 24-30/9/1998.

[ 18$]$ Mostra personale all'Hilton Hotel di Nicosia, Cipro, 22-26/02/2006.

[19] La mostra è stata organizzata dal Centro di Documentazione e Videocomunicazione DOMUVICO, responsabile scientifica Arch. Renata Bizzotto, Roma, I I- | 8/ I |/20 I3. Ė un percorso espositivo che mette a confronto "ottiche diverse, culture, interessi e ricerche sugli spazi dove l'uomo vive, lavora"', come si legge nella brochure.

[20] Copia risalente al XII-XIII secolo di una carta romana del territorio dell'impero romano, composta da I I pergamene accostabili. II suo essere una rappresentazione topologica la rende facilmente utilizzabile per i viaggiatori.

[2 I] Una profonda gratitudine va indirizzata a Mario Docci e Renata Bizzotto che ci hanno permesso di intraprendere questo percorso di conoscenza, precisando che i temi e le questioni messe in campo dalle opere di Cascarano sono innumerevoli per quantità e qualità delle rappresentazioni.

\section{Riferimenti bibliografici}

Basso Peressut Luca (a cura di). (1997). Stanze della meraviglia. I musei della natura tra storia e progetto. Bologna: Clueb.

Calvino Italo (2019). Le città invisibili. Milano: Mondadori.

De Fiore Gaspare (a cura di). (2002). Gaspare de Fiore disegni, incisioni, progetti. Roma: Kappa.

De Zambotti Armando, Pivetti Franco (a cura di). (1997). Albrecht Dürer viaggiatore nel continente dell'arte un itinerario europeo a cinque secoli dal passaggio in Italia 1494-95 / 1994-95. Atti del Convegno, Arco (Tn), 2, 3/I2/1995. Trento: Improvisazione Prima.

Ohnheiser Danièle (2006). Voyage à Rome avec Montaigne, Stendhal, Chateaubriand, Goethe.... Roma: Palombi Editori.

Vagnetti Luigi (1955). II Disegno dal vero. Genova:Vitali e Ghianda.

Vigna Elio (200 I). Il disegno dell'anima. Dialogo tra un maestro e l'allievo. Genova: Sagep.

\section{Autori}

Antonio Conte, Università degli Studi della Basilicata, antonio.conte@unibas.it Ivana Passamani, Università degli Studi di Brescia, ivana.passamani@unibs.it

Per citare questo capitolo: Conte Antonio, Passamani Ivana (2020). Disegno sempre anche quando penso. I luoghi e l'architettura attraverso visioni inedite di Cascarano/l Always draw even when I think. Places and architecture through unpublished Cascarano visions. In Arena A., Arena M., Brandolino R.G., Colistra D., Ginex G., Mediati D., Nucifora S., Raffa P. (a cura di). Connettere. Un disegno per annodare e tessere. Atti del $42^{\circ}$ Convegno Internazionale dei Docenti delle Discipline della Rappresentazione/Connecting. Drawing for weaving relationships. Proceedings of the 42th International Conference of Representation Disciplines Teachers. Milano: FrancoAngeli, pp. 3155-3182 


\title{
I Always Draw even when IThink. Places and Architecture through Unpublished Cascarano Visions
}

\author{
Antonio Conte \\ Ivana Passamani
}

\section{Abstract}

"I always draw even when I think". The paper has a difficult task: to present, in a few pages, the experience, the creative, poetic and technical activity of an architect, bypassing the simple cataloging of his works. Michele Cascarano lives by drawing and, at the same time, draws by living places, cities and architectures, testifying a theoretical thought. Investigating this silent figure allows to open a cross-section on some aspects of the history of the profession of architect in the second half of the twentieth century. The paper highlights characters and methodological issues of the very close intertwining between theoretical foundations and research in architecture, through its representation: drawings, watercolors that fix on paper visited or imagined cities, between memory and oblivion of timeless works. Cascarano's work shows the existence of an individual research intended as a collective work of being an architect, that uses the art of drawing as an original experience of the reality and of the world. From this reason, he leads to the energy and the representative strength to draw tendentious architectures, placed between survey, memory and design. Thus, the building activity is the art-work of architectural creation, and it amounts to a habitable world that is not influenced by ruins of time or negligence of men.

The viewer is involved in the vertiginous adventure of a thought: the design of cities mosaics is the nexus, the sense of existence. To think by drawing, to understand some realities of the world and make them ideal $[I]$.

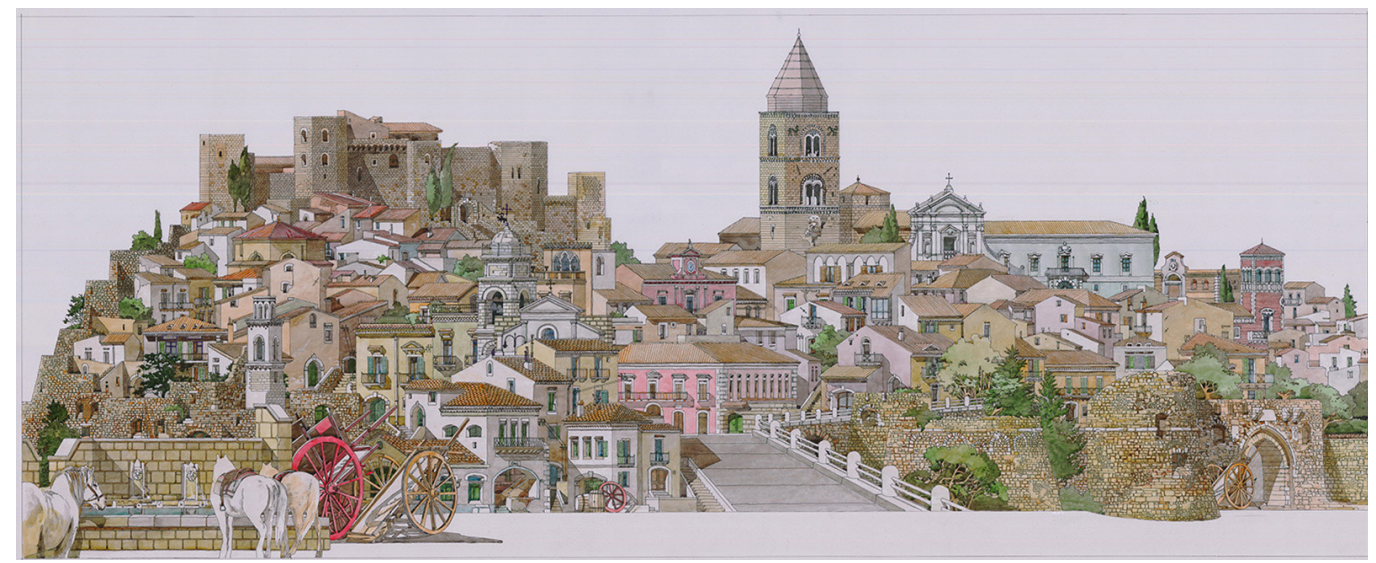




\section{The reason of a choice}

"I always draw even when I write": so, says Günter Grass [2], and adds that "writing and drawing are related". These reflections gave me the opportunity to think that, in the case of Michele Cascarano, the paraphrase "I always draw even when I think", with which we titled this paper, could be valid.

It is difficult to present in a small space an architect's experience, creative and technical activity and his poetics: above all it is not easy to go beyond the simple description of the works to outline what we can also call a "soul design" [3]. It pervades his entire production, reproduces themes and issues addressed in the work but, at the same time, investigates suggestions and many things he meets, which deserve attention until they totally invade spaces and surfaces of all his home-studio, to which we will return later.

He lives by drawing and drawing by living places and architectures. Our interest is therefore motivated by the fact that investigating this silent figure allows us to open a cross-section on some aspects of the history of the profession of the architect in the second half of the twentieth century; at the same time it offers the starting point both to discuss the meaning that this profession can have in the 2 I st century, and to ask ourselves what value and utility could have, in education, traditional representation languages such as drawing, painting techniques, models and maquette.

The sensitivity of the eye that captures and the industriousness and mastery of the hand that translates into signs, backgrounds or cutouts for the construction of models can still be objectives on which to work with young people who approach this profession?

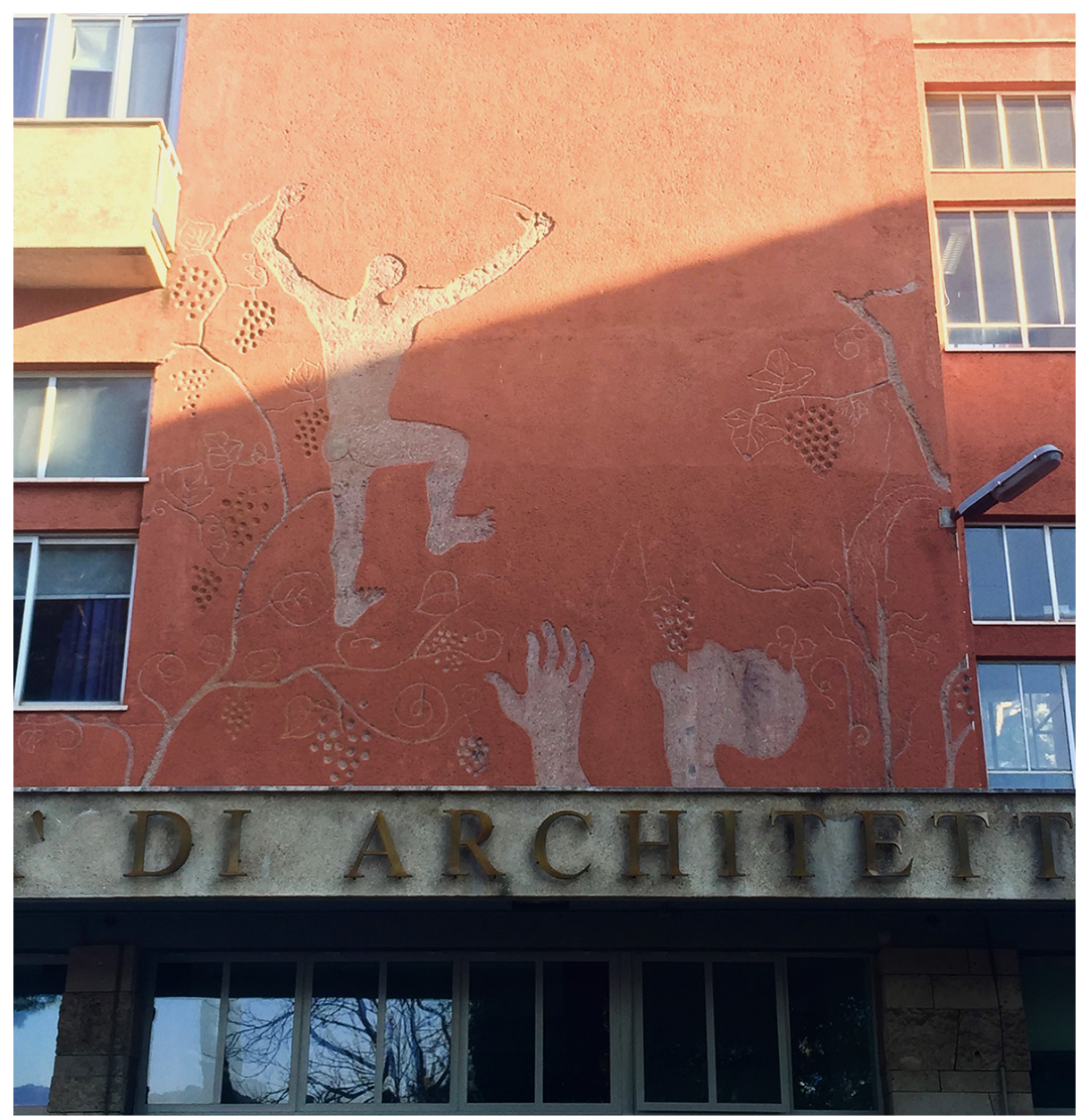




\section{Biographical notes: education in the context of the School of Architecture in Rome of the late twentieth century}

The training course of Michele Cascarano [4] allows us to outline a cross-section on the Roman cultural complexity related to architecture in the "60. On his way, he meets teachers such as Gianluigi Nervi with his assistants Antonio Michetti [5] and Sergio Musmeci [6], and architects who wrote the history of twentieth century architecture: Enrico del Debbio [7], then Director of the Institute of Drawing and Survey of Monuments of the Faculty of Architecture (fig. I), and Leonardo Benevolo, young holder of the chair of History and Styles of Architecture.

The meeting with the Drawing must have influenced his career and consequently his professional and expressive future. In particular, the teachings of architects, then young assistants Gaspare De Fiore [8] and Angelo Marinucci [9], who taught extra-university courses about watercolors that Cascarano attended with other university mates, or Luigi Vagnetti [ I0] who in the course of Drawing from Real trained young students to Drawing "Both as a means of expression, both as a work tool, and finally as a very effective aid in the study of other artistic disciplines" [Vagnetti 1955, p. 5]. In the didactic text on which Cascarano has certainly studied, Vagnetti states: "It is that of Drawing from Real, the only way to simultaneously exercise hand, brain and heart [...]; it is the most effective way to consider simultaneously the obvious opportunity of the analyzes and the absolute necessity of the synthesis; finally, it is the only way to achieve the explication of a complete and independent personality, going through the transitory stages of objective investigation; which then allow maximum freedom of subjective expression, not subordinate to any convention but only aware of the true reality of things" [Vagnetti 1955, pp. 8, 9].

In these words, the claim of Drawing is evident as a way of knowing and taking possession of reality and then representing it in a subjective way with creative freedom: so much that "Painters, Sculptors and Architects" are commonly called "Masters of Drawing" [Vagnetti 1955, p. 5].

Certainly, strong of these teachings, after graduation he enters Valle studio [I I] where he works all his life as Art Director; at the same time, it does not abandon the university world, carrying out teaching activities [12].

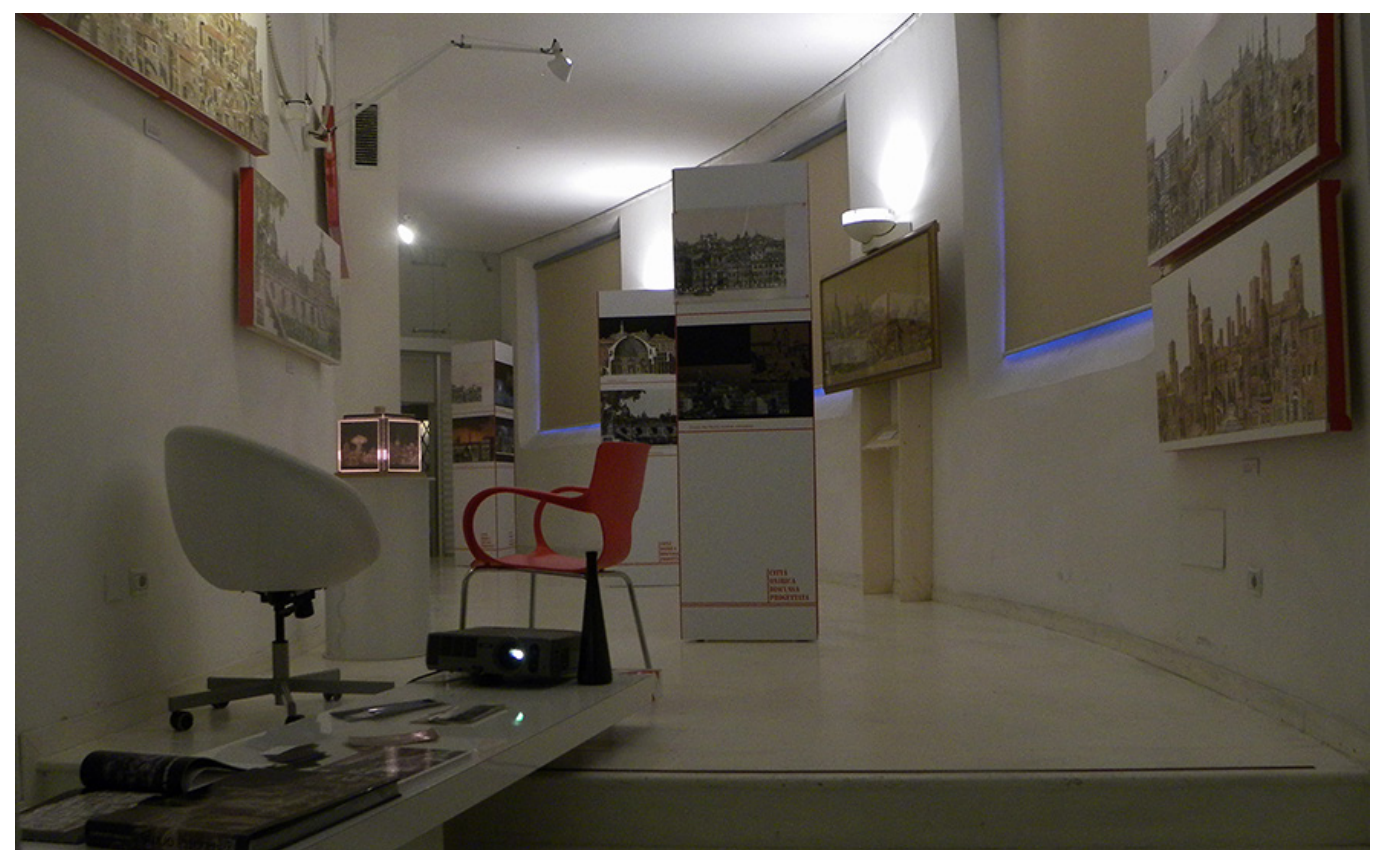


To define Cascarano, therefore, the definition of "Master of Drawing" seems appropriate to us: it is for his teaching activity, it is within his professional activity, where he elaborates complex and very detailed perspectives or meticulous models, it is pursuing with passion and fury his natural need to represent his idea of the city, which is released from the faithful reproduction of the Drawing from the Real in order to diverge in narratives where urban scenes open themselves and building facades become a continuous narration, in a story that it makes dynamic path of the observer.

His long collaboration with Valle studio, very active in international competitions and designs, offers him numerous opportunities to travel the world. It therefore acquires a particular ability to grasp the genius loci and to translate it graphically.

\section{The exhibitions}

Since 1965, the artist has participated in exhibitions in Italy [13] and abroad [14], gaining interest and appreciation.

Some exhibitions titles, reveal the poetic result to the visitor: in "The dream city" [15], for example, quoting Calvino, he underlines that "it is the mood of the beholder that gives the city its shape" [16] to introduce "seven great watercolor works in which urban fantasies, unreal views of real architecture, city skylines seen through a dream, remind of travel diaries" [17]; "The room of dreams" anticipates the sensations communicated by the exhibited works, while "Mediterranean" [18] suggests a cultural-geographical reference area.

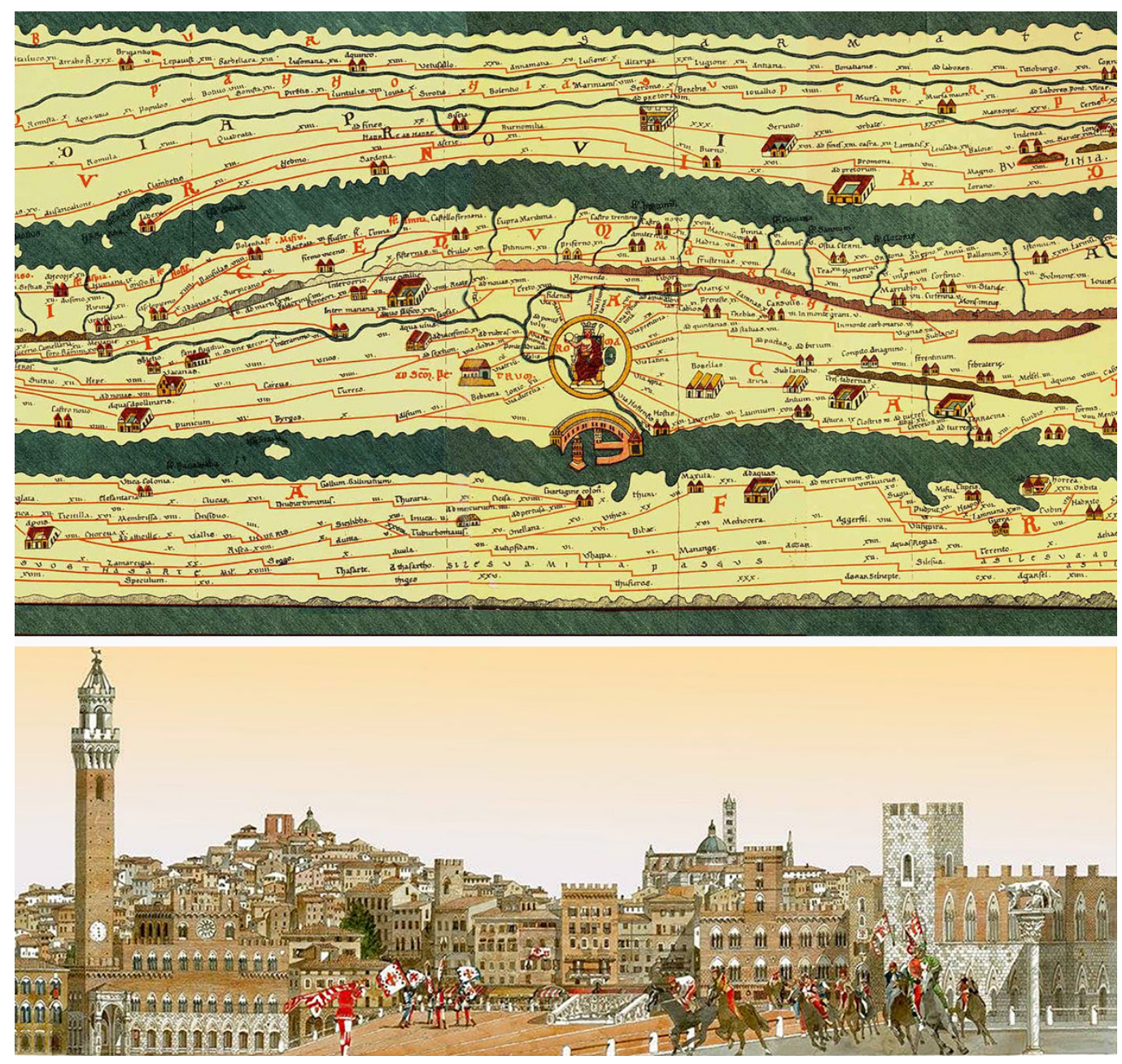


Unlike these initiatives, focused on specific themes, the exhibition "dream city, discussed city, planned city" at Casa dell'Architettura in Rome [19] offers a reflection on the wide and varied production of Cascarano, allowing a knowledge of the architect's multifaceted personality (fig. 2). In fact, large watercolors (also with chromatic variations, sepia or black and white toning) on the city of Rome are exhibited, as well as design models and lamps.

Fig. 4. Above, S. Marco square towards the Basilica, Venezia, Antonio Canal knows as il

Canaletto, pen drawing; below, S. Marco square, Venezia, watercolor by Michele Cascarano (graphics by l. Passamani).
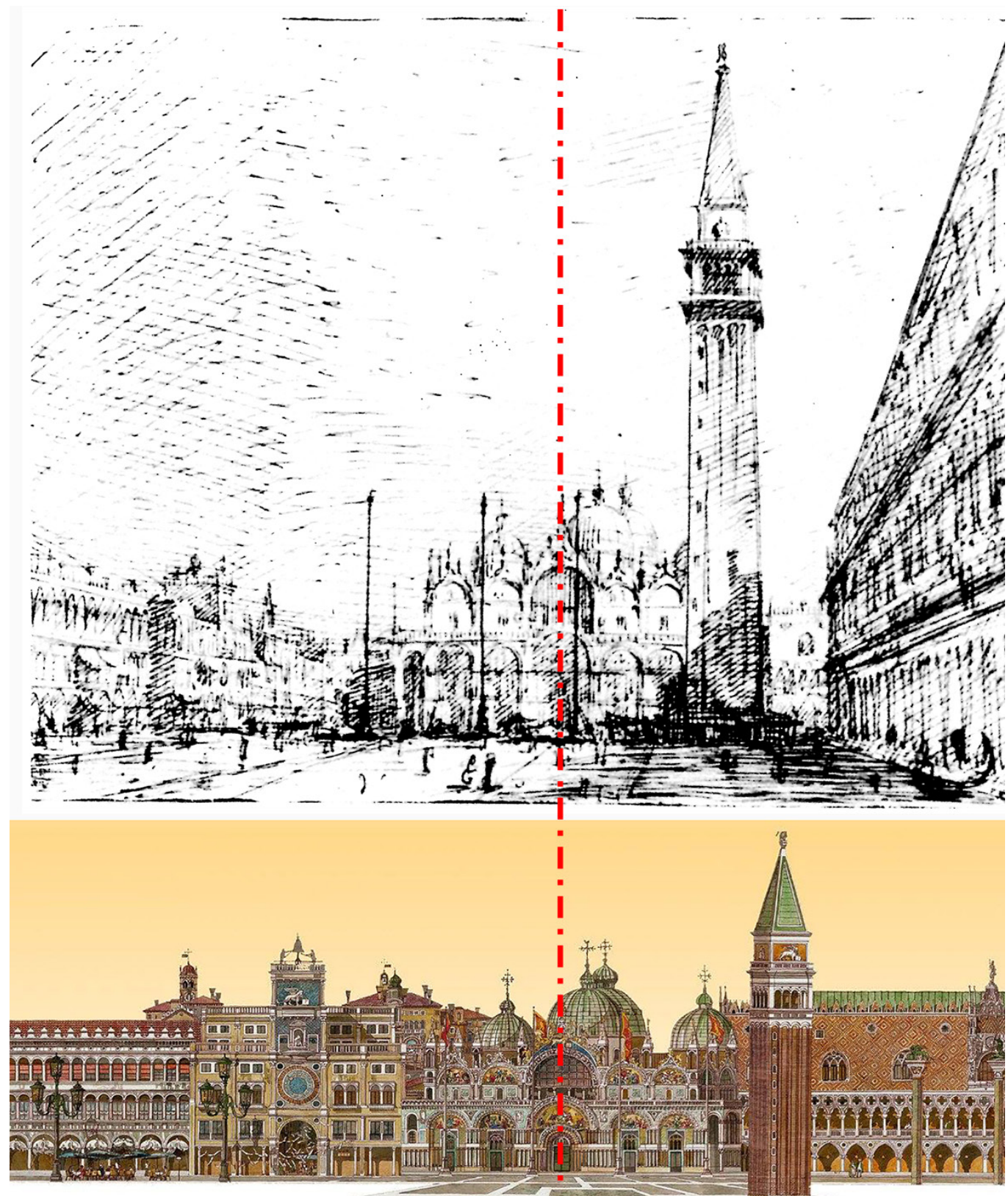

The precedents, the suggestions, the references

Cascarano graphic production allows to identify some famous references, which we mention here with keywords and synthetic considerations, reserving further detailed studies. 


\section{Travel drawings or for the travel}

The large watercolors in which urban fronts of Italian and foreign cities are unfolded in succession, refer to the Tabula Peutingeriana [20] for the similarity of assembling a story of places in sequence; in the case of the ancient map, the places are described on a territorial scale and the story and use are functional to travel, the depictions of cities in the ours are ex post narratives. Both force those who enjoy it to make a virtual and mental journey to the places that the drawing represents (fig. 3).

Inevitable, then, the combination with the drawings produced by the travelers-illustrators key players of several Grand Tours in the 18th and 19th centuries, who underwent the fascination during their journeys along Italy: they left wide-ranging panoramas in which the intent was to fix everything visible.

Surely even Cascarano, traveler for work and passion, does not want to leave anything out: we can recognize its cities and squares by the apparent accuracy with which they are drawn ... but upon careful observation emerges that subjective freedom of expression (evoked by Vagnetti) which transforms landscapes from memory into dreamlike ones: it is surprising, therefore, to immediately recognize a square and then discover that it is not exactly like that ... a healthy surprise, makes us start a critical process (fig. 4), makes us feel architects!

\section{The landscape painters}

In the setting of the points of view of his perspective visions, we can see references to the representations of the landscape painters: Canaletto and Francesco Guardi for the Venetian visions (fig. 5), Filippo Juvara and Gianbattista Piranesi for the urban views of Rome (fig. 6).

Fig. 5. Above, view of Riva degli Schiavoni,Venezia, Francesco Guardi, ink and watercolor; below, view towards S. Marco square Venezia, watercolor
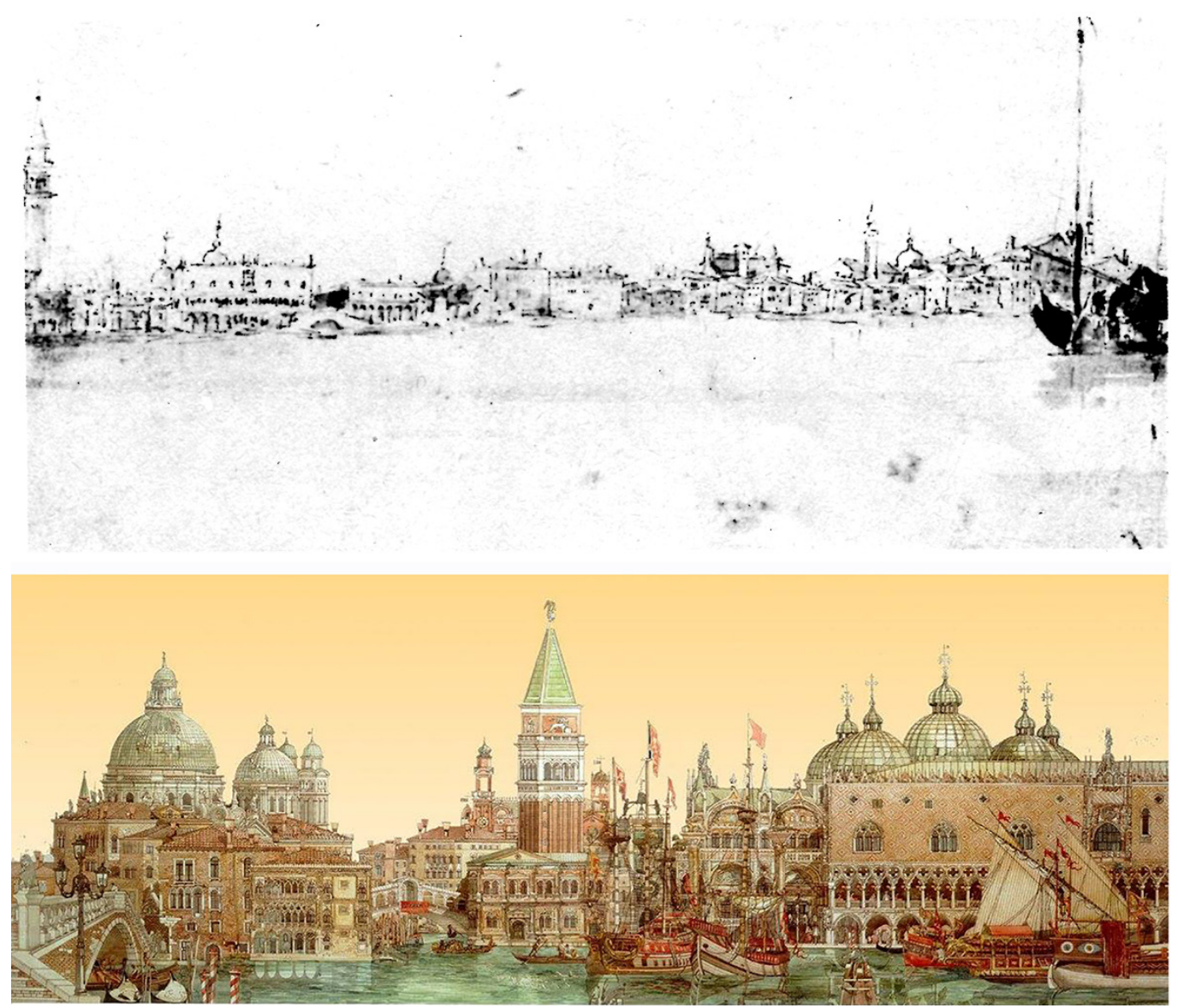
Fig. 6. Above, view of Campidoglio from Aracoeli square, Rom Filippo Juvara, pen and watercolor: below, view of Campidoglio from Aracoeli square, Roma watercolor by Michele Cascarano (graphics I. Passamani).

Fig. 7. Above, Trevi Fountain, Roma, Giambattista Piranesi, enoraving in the center, Trevi Fountain, deterioration, Roma, Gaspare de Fiore, drawing. tion of Tre Cascarano.The graphics Cascarano. The graphics (l. Passamani) relers to the axis 0 the fountain and that of of SS. Vincenzo and Anastasio in Trevi. This facade is the common element Cascarano.
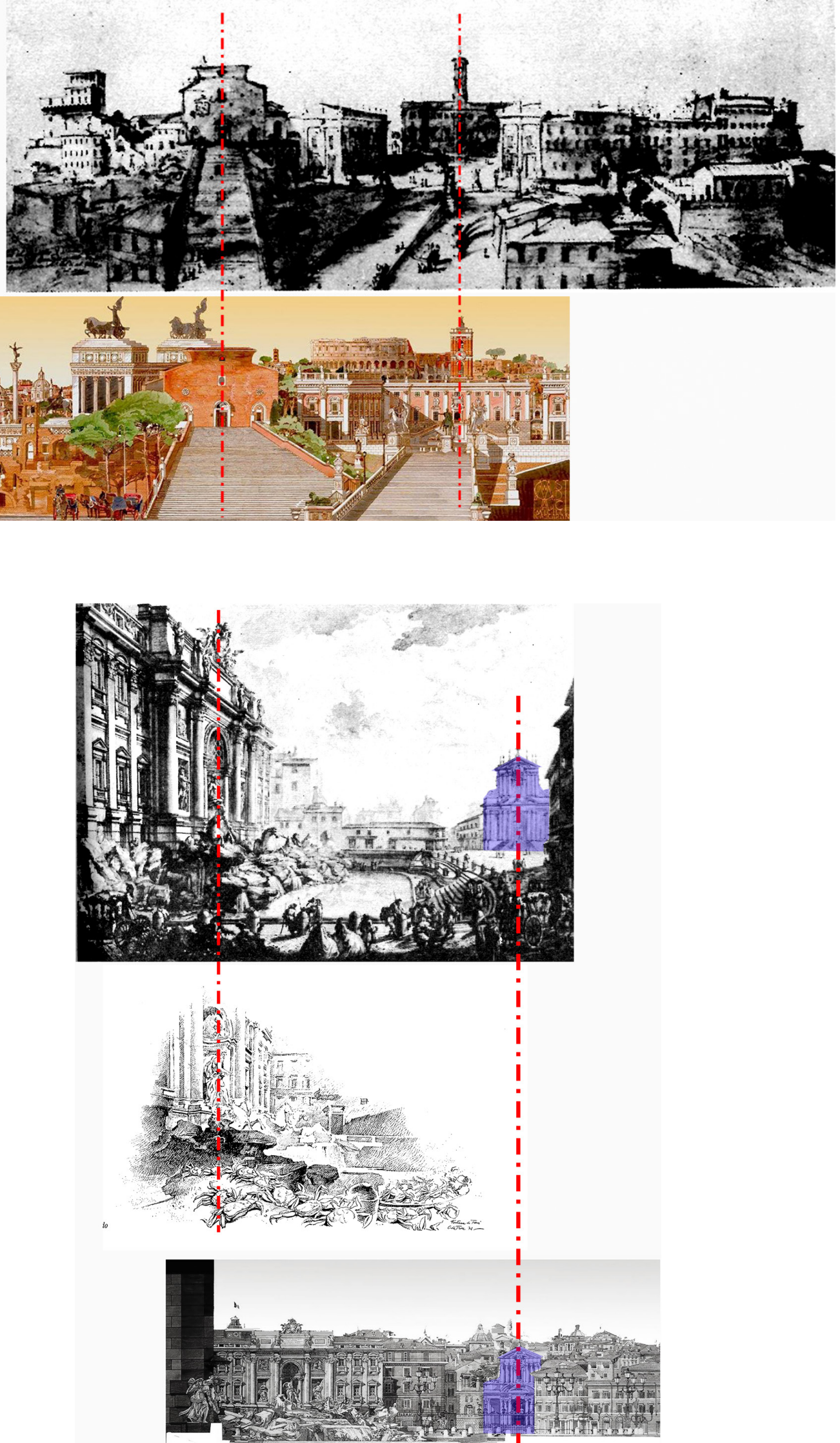
Some of them, which we propose in connection with those of Cascarano, are taken from Vagnetti's text [Vagnetti 1955]: evocations and suggestions, points of view that surely Ours has introjected in the years of his formation.

Illuminating the diachronic assembly of different interpretations of Trevi Fountain: a temporal sequence that starts from the angled view of Piranesi, passes through a drawing by Gaspare de Fiore faithful to the perspective cut, arrives in Cascarano which opens the urban box by placing the fountain on the same level of the picture of the church of SS. Vincenzo and Anastasio (fig. 7).

Fig. 8. The realization of the little theatres, small scenic machines in painted cardboard, alludes to famous quotes such as that of the scene of the 2nd act of Don Giovanni of Mozart. Above a scenery (Arena of Verona, director Franco Zeffirelli), below the small theatre of Don Giovanni of Mozart, watercolor on cardboard by Michele Cascarano (graphics I. Passamani).
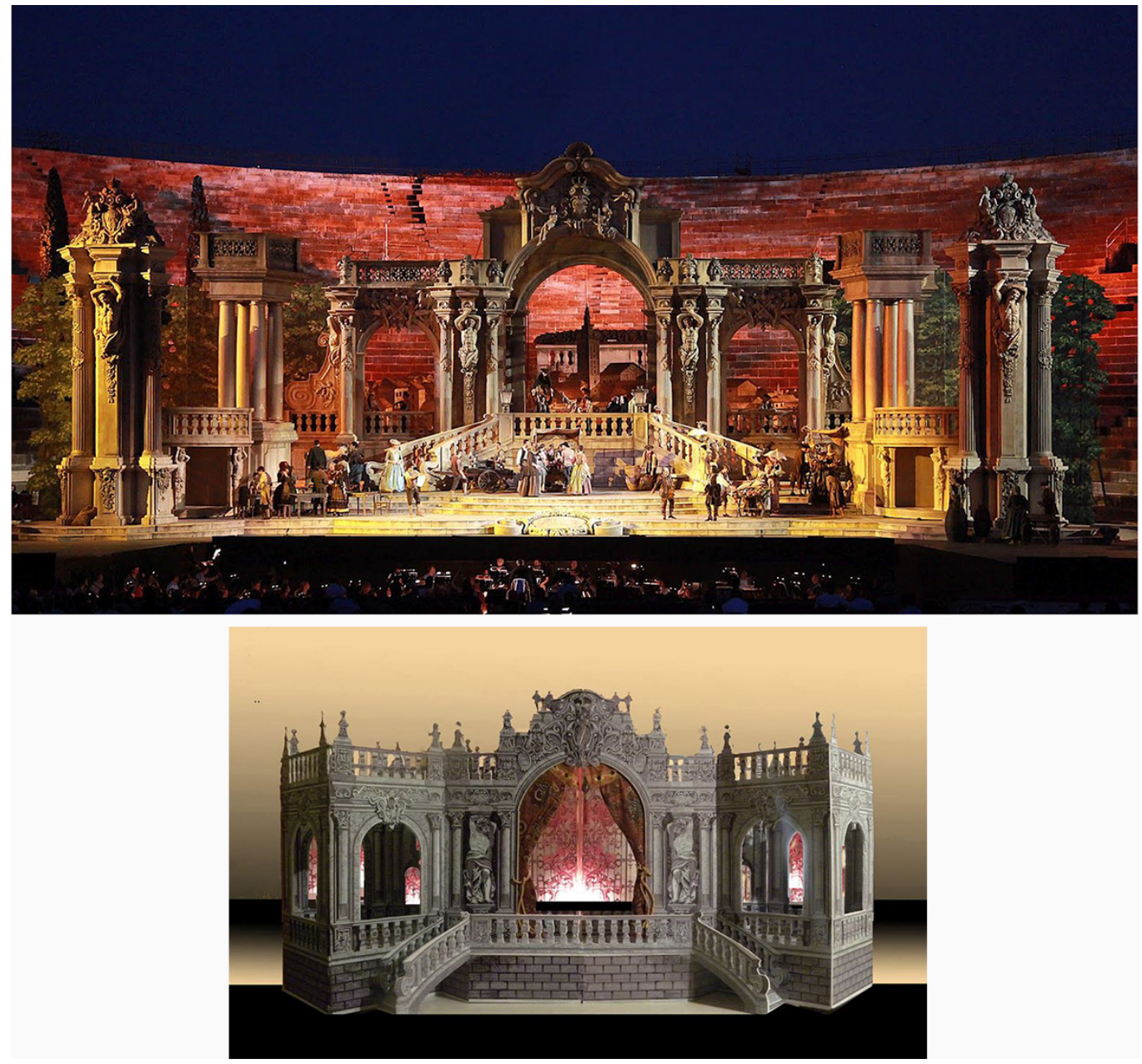

\section{The dream space}

Its private space is dreamlike: as a mirror of itself, as its extension, it gathers its graphic interpretations of external reality alongside concrete objects of the external world and dreamlike constructions, such as the "Travel Theaters" (fig. 8).

It is an anthropomorphic space, characterized by that horror vacui that characterized the ancient Wunderkammern: rooms of wonders that collected, to the delight of the collector of "showing" [Basso Peressut 1997, pag. IX], the collected objects.

Those mirabilia that represent the inner universe of Cascarano (fig. 9). 


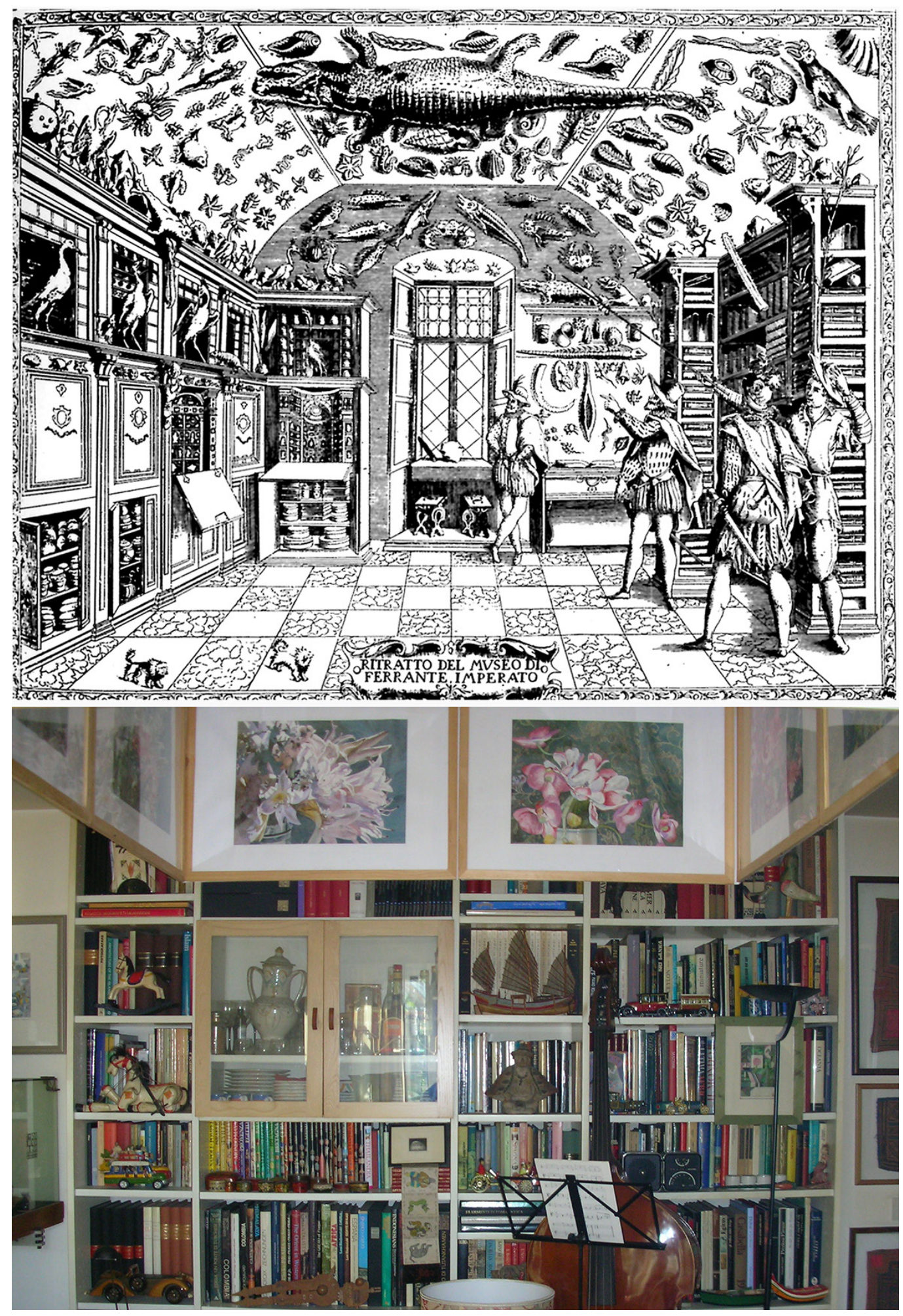




\section{These artifices of places and cities are architectural narrative. With which critical method can you disassemble and reassemble these timeless archi- tectural drawings?}

The considerations collected in this contribution are a form of synthesis of the experience which, by a luckily coincidence, adds to a personal and deep judgment of the figure of one of the many minor Masters who fill the real history of our existence on Earth.

Our complex culture of living the space that we imagine, think, design, build, represents a continuous unveiling of deep intertwining between ideation and thought, imagination and construction of the drawing architecture with the one that was built.

These are unpublished pages which, projected in the contemporary debate on architecture of the second half of the twentieth century and of the new millennium, illuminate with a clearer light the sense of contemporary living in a specific contribution that inserts, in the historical-critical and documentary apparatus of the Community, research trajectories of unexpected dimensions, like the works of many Masters that we don't know at all [2I]. It is my interest to try to highlight characters and methodological issues of the very close intertwining between theoretical foundations and research itself in architecture, through Cascarano's representation and drawings.

A second part, in a personal form, intends to define a real immersion in the hundreds of graphic tables, drawings, real engravings, watercolors, which fix on paper the almost infinite territory of cities visited and imagined, between memory and oblivion of timeless architecture. His work represents and shows the existence of an individual research, at the same time intended as a collective work of being an architect who places in the art of drawing a vision and an original experience of reality.

From this foundation, he brings the energy and the representative strength to draw architectures naturally placed between survey, memory and design.

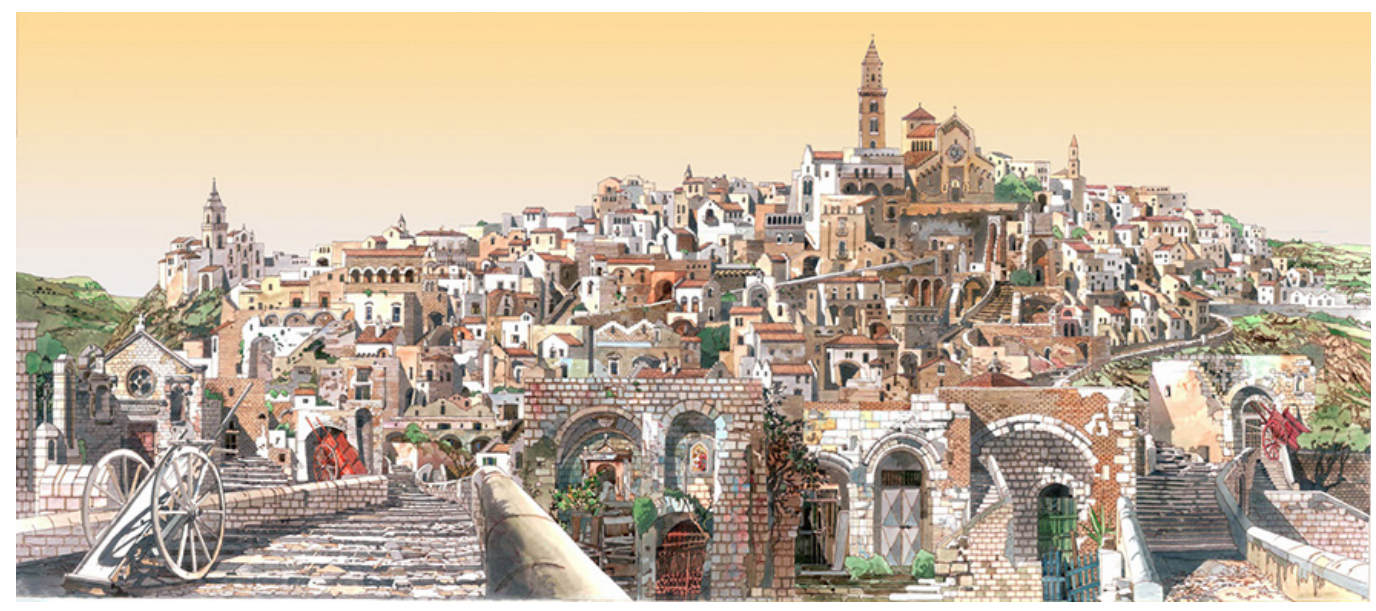

The matter that emerges is a sort of assumption, a vocation to consider the city from an analytical point of view which is a fundamental moment for the development of knowledge and design in the field, although partial, of doing architecture.

The relationship between architecture, rationality and invention represents the reality built in a very concrete way and many drawings of invention, of parts of analogous cities composed with parts and elements of other places, are a unique disciplinary corpus, without which any disciplinary advancement would be partial: it is our responsibility to make it known and live in the time of history. These theoretical and critical assumptions often mean for us the faith, more or less conscious, in the Drawing as a professional practice and didactic experimentation which always represents a sort of shortcut to the design project and to the construction. 


\section{The reduction of architecture reality to complex visions and narratives}

This reduction of architecture reality to complex representations that are visions and narratives of synthesis processes, does not prevent the development of different forms of knowledge and interpretation of places in history, even if only imagined as analogous construction of cities.

Putting ourselves in front of these drawings, we can see, regardless of size, parts and elements, areas and places, the characters of urban facts whose presence is the critical soul that fills Michele's life with Drawing as the "necessary Angel". He has seen Rome, Venice, The Cairo, Barcelona, (fig. I0) countless cities and, with obsessive narrative, traces authentic geographies, identifies the nature of the landscapes, identifies possible configurations even at the cost of visualizing imagined things, transposed into new urban compositions.

These very long city tables, somehow recomposed in a dreamlike fixity, are a dense and continuous plot of stylistically relevant monuments and architectures, which stand as a visionary hope of real beauty. I think he lived this part of his life by drawing, in an obsessive attempt to proceed with the construction of a "hoped-for city", of many cities and to use a series of different elements, connected to each other but whose meaning is precisely in the experience of defining one possible "ideal shape".

The city therefore by its very nature is not a unique creation and can be traced back to basic ideas from thought, to the synthesis of an extraordinary formation. These drawings are presented as an accomplished work of relocation in an eternal fixity of that moment, of that observation carried out in several directions, from real research to beauty. The individuality

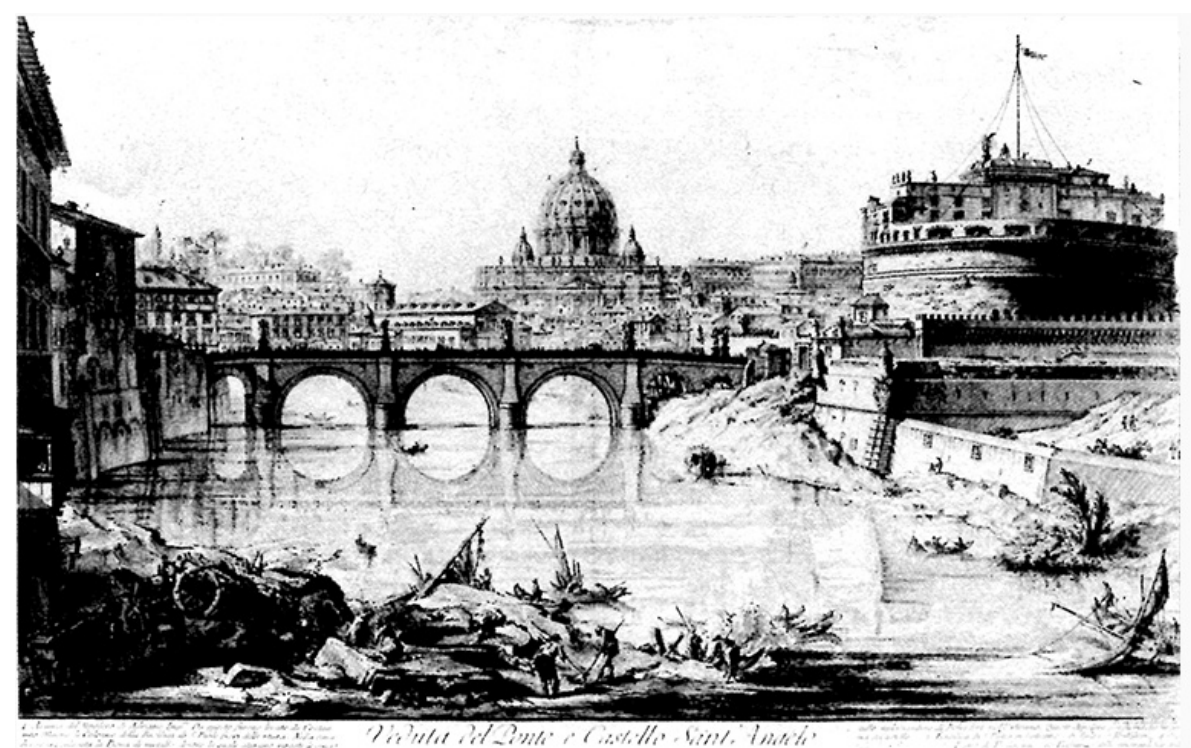

Fig. I I. Above, view of the bridge and of S. Angelo Castle, Gianbattista Piranesi; below view of S. Angelo Castle, Roma, watercolor by Michele Cascarano (graphics by I. Passamani).

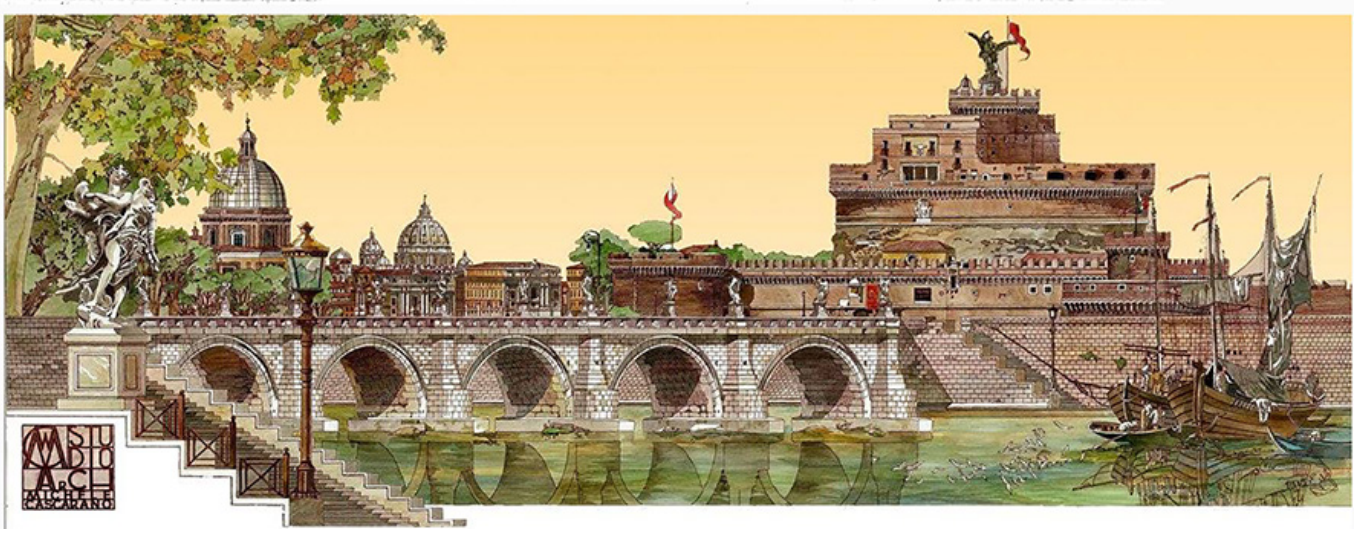


of the parts and of the individual elements represent direct investigations, his speeches and reasoning of existence that from the classification process collect deeper personal poetics. This form of work, of scientific investigation on cities and on their type-morphological characters, presents itself as a vision of methodological approaches aimed at finding progress in history and as a recognition of the autonomy of architecture from human and phenomenological facts.

In this critical process of knowledge, I have tried to clarify an approach to the problem of architectural design that seems to underlie drawings intensely linked to intertwining visions rooted in memory, in dreams, in the imagination and in the illusion of a different, theoretical reality.

I believe it is not necessary to understand the techniques of drawing but to grasp that dialectical intertwining between observed urban reality and elements of architecture traced in the precision of detail, as a deep meaning of every design and construction experience. In all the drawings, from those made by tiny lines to the very fine watercolors, a mastery in the use of the tools of a disciplinary corpus is evident, which aims to the build and to the advance in architecture as a wealth of knowledge and of critical tensions. They represent individuality, urban facts even in an ideal form that we could place timelessly between the drawings of Canaletto and Piranesi (fig. I I).

On the other hand, the analysis and the design apply to the built city, to its architecture and with these it is possible to compare, make formal and theoretical choices.

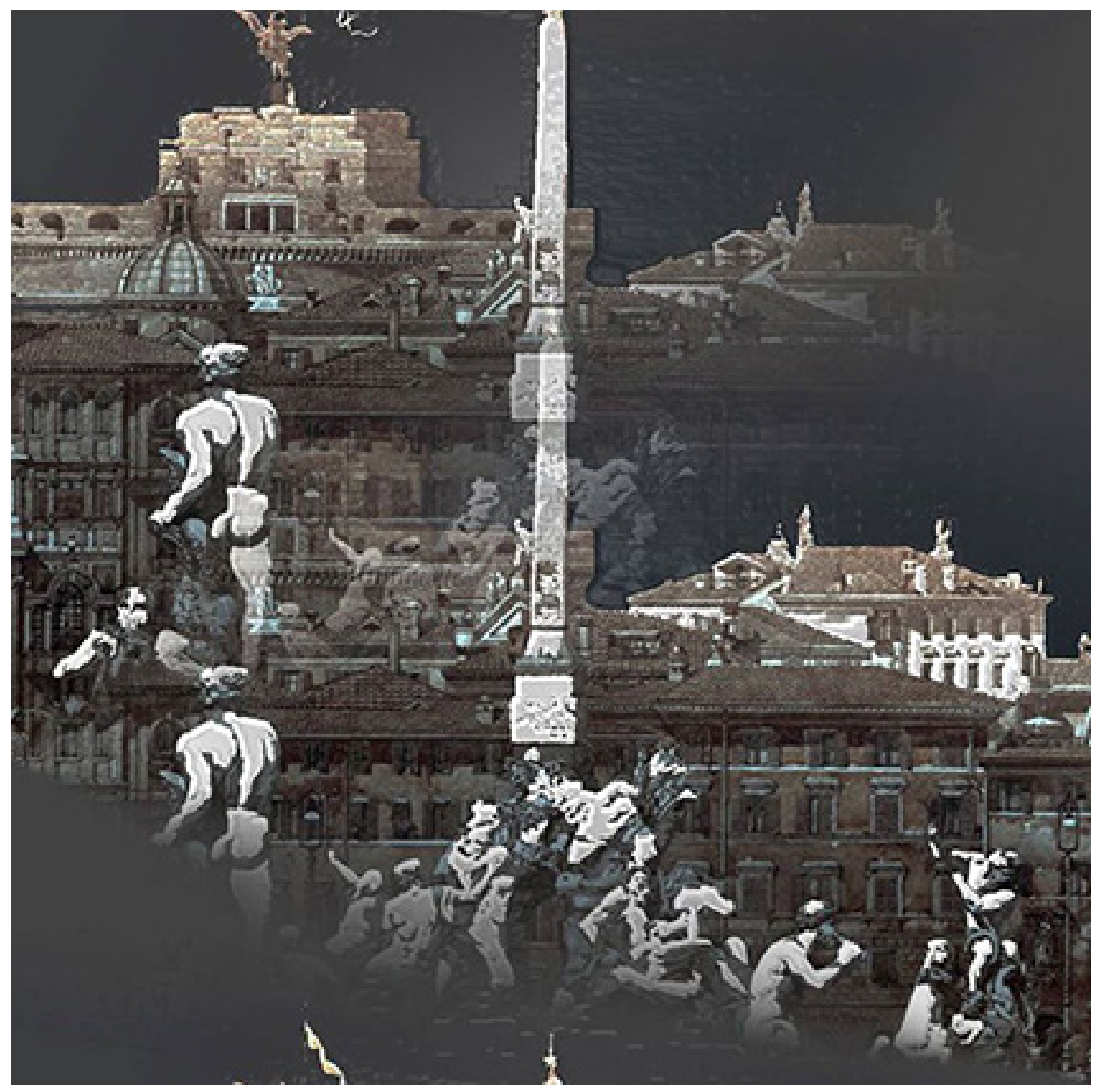


In these works, we recognize this character that binds every thought to the drawing, to the research aimed at making clear the nature of architecture and of the cities in a vision of possible transformations and civil values that invest daily the work of an architect, of failures, of precariousness, the fragility of actions and being able to change things through architecture. My considerations therefore concern a subject expressed in a rational form that underlies adherence to a world view that invests the problem of the meaning of architecture as an ethical and civil sense of humanity. This critical discourse originates from the theoretical role of these experiences to attempt to re-construct a possible scientific discourse on architecture, starting from the quantity of works and drawings that run through the lives of minor Masters, often unknown who present themselves to us.

\section{Conclusions. An exhibition to debate and reflect on the meaning and role of architectural drawing as knowledge and design of reality}

Since the first meeting, it has always been my belief that Michele Cascarano's knowledge represents an important cultural and educational opportunity for the young School of Architecture in Matera: to critically present the work of this great designer, obstinate and incessant, of a life dedicated to the project, it can fill some absences in the history of representation and Drawing as Knowledge.

This critical activity, which starts with many clarifications for the definition of an exhibition of his works, fills me with pride and a sense of responsibility, with a thread of concern for the numerical dimension of the works and for their undisputed and extremely high quality and wealth of contents.

The role played by the knowledge of architecture, of its lifelong practice, outlines a constant practice of the observer, by continually modifying the built environment, constructing a sort of "hoped-for city" in an alternative way, also transforming the ruins into perfectly completed places and catalogs filled with a second nature of existence and beauty.

Among the artifices of this extraordinary stability of the forms of man-made things, of their transience, Michele makes reasonable choices and reserves the right to deliver them for as long duration as possible, offering himself and the world luminous representations of the very meaning of manufacturing: he does this by establishing a stable vision of the cities, of the architectures that make up the fixed scene of the permanence of art in history and over time.

Thus, the act of manufacturing and the art work of architectural creation are forever welded and connected to rediscover and configure a habitable world not subject to the ruins of time and to the neglect of men.

The observer is involved in the dizzying adventure of a thought, whose act of building represented by these city mosaics (fig. 12) is the nexus, the sense of existence, of thinking by drawing to understand some realities of the world.

\section{Notes}

$[I]$ The study activity on the figure of Michele Cascarano was started by both authors of this paper, who share an interest in deepening the many aspects of the architect's design and artistic production. It should be noted that Ivana Passamani is the author of paragraphs $I$ and 2 and Antonio Conte is the author of paragraphs 3 and 4.

[2] Günter Grass (1927-20 I5), writer and poet, sculptor and painter, received the Nobel Prize for literature in 1999.

[3] We like to borrow this definition by citing the title of a text by Elio Vigna, 200 I.

[4] He was born in Melfi (Potenza) on February 10, 1936. After the end of the war his family moved to Rome, the city where he still lives and works. Here he attended high school, enrolling in 1954 at the Faculty of Architecture at "La Sapienza" University. He graduated with honors in 1961.

[5] Who, like me, was lucky enough to know Eng. Antonio Michetti (1927-2010) and to attend his lessons in "Building Technique", agrees that he was "the most loved between Roman engineers". It is therefore not surprising to receive the Honoris Causa Degree in Architecture (La Sapienza, Rome, 03/03/2003): he was deeply an architect, as evidenced by his statement "The first design is not static, but architectural". In his long professional career Antonio Michetti has always worked 
in line with the lesson of his master Pier Luigi Nervi, contributing to the definition of the image of contemporary Italian architecture.

[6] Sergio Musmeci (1926-198I), structural engineer, began his activity with Morandi and Nervi; his is the structural project of the Swimming Stadium at the Foro Italico in Rome (1959), whose architectural project is signed by Del Debbio and Vitellozzi.

[7] Enrico del Debbio ( 89 | - 1973) signs the project of the Faculty of Architecture of Valle Giulia in Rome.

[8] Gaspare de Fiore ( $1926-201$ I), architect and teacher, illustrator with Jacovitti and others of the magazine "II Vittorioso" in the 1950s.

[9] Angelo Marinucci (1909-1994) is an architect, teacher and painter. Free lecturer at La Sapienza, he collaborates with Vagnetti on the live Drawing course.

[10] Luigi Vagnetti (1915-1980), architecture and drawing theorist, university professor and designer, also makes a fundamental scientific contribution in the field of architectural survey, which he considers to be connected in an essential way to reality representation.

[I I] Cesare Valle (1902-2000), architect, is a professor of town planning at the Faculty of Engineering of Sapienza; he is the author of numerous architectural projects and town plans.

[12] He teaches in drawing courses, watercolor technique and chromatism in architecture.

[13] Roma, Ovindoli, Rocca di Mezzo, Assisi, Civitavecchia.

[14]Tokio, Cipro.

[15] Exhibition at Temple Gallery, Roma 29/9-15/10 1998

[16] Is Zemrude city. Italo Calvino, 2019, Cities and eyes.

[17] In Roma C'è - Arte 24-30/9/1998.

[18] Personal exhibition at Hilton Hotel di Nicosia, Cipro, 22-26/02/2006.

[19]The exhibition was organized by the Documentation and Video Communication Center DOMUVICO, scientific manager Arch. Renata Bizzotto, Rome, I I- | 8/ I |/20 I3. It is an exhibition path that compares "different perspectives, cultures, interests and researches on the spaces where man lives, works", as stated in the brochure.

[20] Copy dating back to the XII-XIII century of a Roman map of Roman Empire territory, made up of I I matching parchments. Its being a topological representation makes it easily usable for travelers.

[2I] A deep gratitude goes to Mario Docci and Renata Bizzotto who have allowed us to undertake this path of knowledge, specifying that themes and issues brought into play by Cascarano's works are innumerable for quantity and quality of the representations.

\section{References}

Basso Peressut Luca (a cura di). (1997). Stanze della meraviglia. I musei della natura tra storia e progetto. Bologna: Clueb.

Calvino Italo (2019). Le città invisibili. Milano: Mondadori.

De Fiore Gaspare (a cura di). (2002). Gaspare de Fiore disegni, incisioni, progetti. Roma: Kappa.

De Zambotti Armando, Pivetti Franco (a cura di). (1997). Albrecht Dürer viaggiatore nel continente dell'arte un itinerario europeo a cinque secoli dal passaggio in Italia 1494-95 / 1994-95. Atti del Convegno, Arco (Tn), 2, 3/ I 2/I 995. Trento: Improvvisazione Prima.

Ohnheiser Danièle (2006). Voyage à Rome avec Montaigne, Stendhal, Chateaubriand, Goethe.... Roma: Palombi Editori.

Vagnetti Luigi (1955). /l Disegno dal vero. Genova:Vitali e Ghianda.

Vigna Elio (200 I). Il disegno dell'anima. Dialogo tra un maestro e l'allievo. Genova: Sagep.

\footnotetext{
Authors

Antonio Conte, Università degli Studi della Basilicata, antonio.conte@unibas.it Ivana Passamani, Università degli Studi di Brescia, ivana.passamani@unibs.it

To cite this chapter. Conte Antonio, Passamani Ivana (2020). Disegno sempre anche quando penso. I luoghi e l'architettura attraverso visioni inedite di Cascarano/l Always draw even when I think. Places and architecture through unpublished Cascarano visions. In Arena A., Arena M., Brandolino R.G., Colistra D., Ginex G., Mediati D., Nucifora S., Raffa P. (a cura di). Connettere. Un disegno per annodare e tessere. Atti del $42^{\circ}$
Convegno Internazionale dei Docenti delle Discipline della Rappresentazione/Connecting. Drawing for weaving relationships. Proceedings of the 42 th International Conference of Representation Disciplines Teachers. Milano: FrancoAngeli, pp. 3155-3|82.
} 